\title{
Avaliação da ventilação mecânica utilizada em unidade de terapia intensiva pediátrica e seus fatores de risco: em busca de uma melhor prática ventilatória
}

Dissertação apresentada à Faculdade de Medicina da Universidade de São Paulo para a obtenção do título de Mestre em Ciências

Área de concentração: Pediatria.

Orientador: Prof. Dr. Eduardo Juan Troster

São Paulo 
Dados Internacionais de Catalogação na Publicação (CIP)

Preparada pela Biblioteca da

Faculdade de Medicina da Universidade de São Paulo

Creprodução autorizada pelo autor

Silva, Dáfne Cardoso Bourguignon da

Avaliação da ventilação mecânica utilizada em unidade de terapia intensiva pediátrica e seus fatores de risco : em busca de uma melhor prática ventilatória / Dáfne Cardoso Bourguignon da Silva. -- São Paulo, 2009.

Dissertação(mestrado)--Faculdade de Medicina da Universidade de São Paulo. Departamento de Pediatria.

Área de concentração: Pediatria.

Orientador: Eduardo Juan Troster.

Descritores: 1.Ventilação pulmonar 2.Unidades de terapia intensiva 3.Fatores de risco 4.Estudos epidemiológicos 5.Criança

USP/FM/SBD-336/09 


\section{Dedicatória}

Ao meu filho, Hugo, cuja gestação e nascimento coincidiram com o término desta tese. Um novo ciclo de vida que se inicia para mim, cheio de amor, com grandes expectativas e com a sensação de ter feito o meu melhor... 


\section{Agradecimentos Especiais}

Agradeço ao meu "Querido Chefinho", meu orientador Dr. Eduardo Juan Troster, com quem entendi que o conhecimento teórico não era tudo, que deveria haver não apenas desejo de ensinar e aprender, mas o de se fazer o correto, de se preocupar com o próximo e ser ético. Orientador sempre presente (mesmo quando eu não estava tão presente assim...), que respeitou o meu tempo quando eu precisei de um - e me pôs para trabalhar quando foi hora de voltar. Não só me deu chances de escrever capítulos de livros e artigos, mas principalmente, me ensinou a dar os meus próprios passos na pesquisa. Dizem que cada orientando tem o orientador que merece e vice-versa. Bem, eu tenho muito orgulho em ter sido sua orientanda...

Agradeço aos meus pais, Olívio e Cândida, pelo amor, compreensão e pelos maiores bens que puderam me dar: educação e moral. Que eu possa ter a mesma nobreza de caráter ao criar meu filho - e a mesma capacidade de amá-lo...

Agradeço a meu marido, Cléber, a quem devo tantos momentos alegres e o apoio nos momentos difíceis; companheiro que trilha comigo os mesmos caminhos, e que agora segue ao meu lado para o desafio de sermos bons pais. 


\section{Agradecimentos}

A todos aqueles que contribuíram, de alguma forma, para a realização deste trabalho.

A tantos professores amigos, incentivadores, sem os quais eu nunca teria seguido a vida acadêmica. Agradeço especialmente à Dra. Filomena Eurídice Carvalho de Alencar, por ter acreditado no meu potencial e ter aberto mão da minha companhia e me colocado no rumo certo. Mais que uma preceptora, minha amiga.

À Raquel Belmino de Souza, que compartilhou comigo não só a residência médica, mas o anseio de melhorar o que fazíamos em prol de nossos pequenos pacientes...

À Audrey Rie Ogawa Shibata, companheira de plantões, dúvidas e certezas, sem a qual este trabalho nunca teria sido feito.

Ao Dr. Alexandre Archanjo Ferraro, pelo apoio e pelas orientações referentes à epidemiologia e estatística.

Ao Dr. Antônio Sérgio Petrilli, por sua presença certa e pelas oportunidades que se descortinam.

A todos os meus amigos pelas palavras de incentivo, pela troca de conhecimento, pelo apoio emocional e pelos momentos de alegria, em especial às amigas Fernanda Maria Wanderley Freire e Francyanne Campelo Vasconcelos.

Agradeço também àqueles que trabalharam e permitiram que este projeto se concretizasse: à equipe da UTIP do Instituto da Criança, por sua enorme dedicação no tratamento de crianças tão gravemente enfermas; à Mariza, funcionária da biblioteca do Instituto da Criança, por sua ajuda sempre tão solícita; à equipe do arquivo médico (SAME), e a Adriana e Solange (secretárias da pós-graduação).

Em especial, agradeço aos pequenos pacientes internados que participaram deste estudo, sem os quais o mesmo não teria sido possível.

A todos, meus sinceros agradecimentos. 


\section{Resumo}

\section{Avaliação da ventilação mecânica utilizada em unidade de terapia intensiva pediátrica e seus fatores de risco: em busca de uma melhor prática ventilatória.}

Apesar da necessidade de ventilação pulmonar mecânica (VPM) ser uma das principais indicações de internação em unidade de terapia intensiva pediátrica (UTIP), há poucos estudos epidemiológicos sobre VPM em crianças, nenhum no Brasil. Fazse necessário descrever quais os modos ventilatórios utilizados em nosso meio, para estabelecer qual nosso padrão de cuidado. Dos 241 pacientes admitidos na UTIP do Instituto da Criança entre 01/10/2005 e 31/03/2006, 35,7\% foram submetidos à VPM por mais de 24 horas $(n=86)$. Trinta e sete foram excluídos da análise. Analisaram-se dados de 49 pacientes. A principal indicação de VPM foi insuficiência respiratória aguda. O modo ventilatório de escolha foi à pressão $(n=48)$. A estratégia de ventilação protetora foi subutilizada. São analisados ainda fatores de risco para mortalidade e tempo de VPM.

Descritores: insuficiência respiratória, ventilação mecânica, fatores de risco, unidades de terapia intensiva, pediatria. 


\section{Abstract}

Evaluation of mechanical ventilation used in a Pediatric Intensive Care Unit and its risk factors: searching for a better ventilatory practice.

Mechanical ventilation (MV) is a major admission criteria to pediatric intensive care unit (PICU). Despite of that, there is just a few epidemiologic studies about it in children, and none in Brazil. It's necessary to describe which ventilatory modes are employed in our daily practice, in order to establish our standard of care. 86 out of 241 patients, admitted to "Instituto da Criança" PICU from 10/01/2005 to 03/31/2006, were submitted to MV for 24 hours or more. Thirty seven met exclusion criteria. Data from 49 patients were analyzed. Major indication to MV was acute respiratory failure. Pressure ventilatory modes were used. Protective lung ventilation was underused. Analyses of risk factors for mortality and days of MV were also performed.

Keywords: respiratory insufficiency, ventilation artificial, risk factors, intensive care units, pediatrics. 


\section{Lista de ilustrações}

\begin{tabular}{l|c|}
\hline Quadro 1 - Identificação dos pacientes incluídos no estudo & 17 \\
\hline Gráfico 1 - Indicação da ventilação pulmonar mecânica & 18 \\
\hline Gráfico 2 - Modalidades ventilatórias utilizadas na população & 19 \\
\hline estudada & 21 \\
\hline Gráfico 3 - Volumes correntes utilizados nos pacientes & 23 \\
\hline estudados, ao início e após 48 horas de ventilação mecânica & \\
\hline Gráfico 4 - Distribuição dos pacientes estudados quanto à & 24 \\
\hline relação PaO2/FiO 2 & \\
\hline Gráfico 5 - Distribuição dos pacientes estudados quanto à & \\
\hline pressão arterial de gás carbônico $\left(P_{2} \mathrm{PaCO}_{2}\right)$, ao início e após 48 & \\
\hline horas de ventilação mecânica
\end{tabular}




\section{Lista de tabelas}

Tabela 1 - Parâmetros ventilatórios, dados gasométricos e disfunção de órgãos

Tabela 2 - Pressões inspiratórias utilizadas nos pacientes estudados, ao início e após 48 horas de ventilação mecânica

Tabela 3 - Fatores de risco para mortalidade em 28 dias

Tabela 4 - Fatores de risco para mortalidade e duração de

ventilação mecânica superior a sete dias, com suas respectivas razões de chance ("odds ratio") 


\section{Lista de abreviaturas, siglas e símbolos}

A/C - (Ventilação) Assistido / Controlada

Cm - Centímetro

Curva PxV - Curva Pressão versus Volume

EUA - Estados Unidos da América

$\mathrm{FiO}_{2}$ - Fração Inspirada de Oxigênio

FR - Freqüência Respiratória

$\mathrm{H}-\mathrm{Hora}$

$\mathrm{H}_{2} \mathrm{O}$ - Água

HC-FMUSP - Hospital das Clínicas da Faculdade de Medicina da Universidade de São Paulo

HFOV - Ventilação Oscilatória de Alta Frequência

IGMVC - "International Group of Mechanical Ventilation in Children"

10 - Índice de oxigenação

Kg - Quilograma

LPA - Lesão Pulmonar Aguda

$\mathrm{M}^{2}$ - Metro quadrado

MI - Mililitro

$\mathrm{mmHg}$ - Milímetros de mercúrio

NFkb - Fator nuclear kappa b

OMS - Organização Mundial de Saúde

P - nível de significância estatística: probabilidade de erro envolvida em aceitar

o resultado observado como válido 
PALISI- "Pediatric Acute Lung Injury and Sepsis Investigators"

$\mathrm{PaCO}_{2}$ - Pressão arterial de gás carbônico

$\mathrm{PaO}_{2}$ - Pressão arterial de oxigênio

PCV - Ventilação pressão-controlada

PEEP - Pressão positiva ao final da expiração

PELOD - "Paediatric Logistic Organ Dysfunction"

pH - Potencial hidrogeniônico, grandeza que indica a acidez, neutralidade ou alcalinidade de uma solução líquida (no caso, o sangue).

PmVA - Pressão média de vias aéreas

PRVC - (ventilação) pressão-regulada e volume-controlada

PRVS - (ventilação) pressão-regulada e volume de suporte

PS - Pressão de suporte

Rx - Radiografia

SDRA - Síndrome do Desconforto Respiratório Agudo

SIMV - Ventilação Mandatória Intermitente Sincronizada

SUS - Serviço Único de Saúde

TGI - Insuflação Transtraqueal de Gases

UTI - Unidade de Terapia Intensiva

UTIP - Unidade de Terapia Intensiva Pediátrica

VILI - Lesão Pulmonar Induzida pelo Ventilador

VPM - Ventilação Pulmonar Mecânica

VTPC - "Volume-target, pressure controlled (ventilation)" 


\section{Sumário}

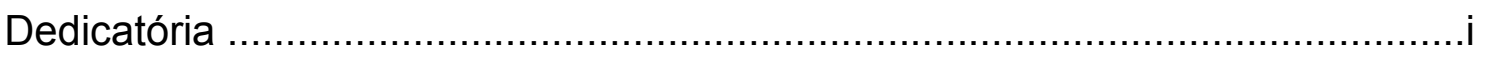

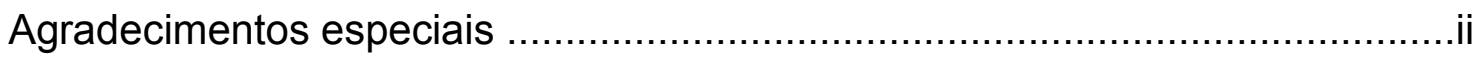

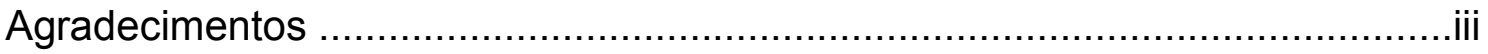

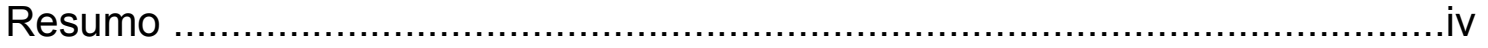

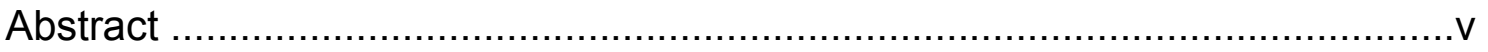

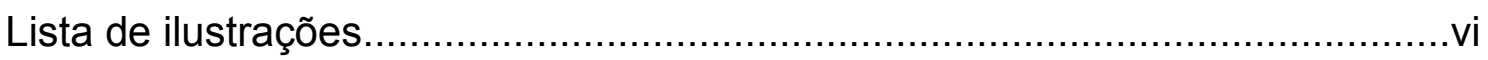

Lista de tabelas............................................................................ vii

Lista de abreviaturas, siglas e símbolos.....................................................iii

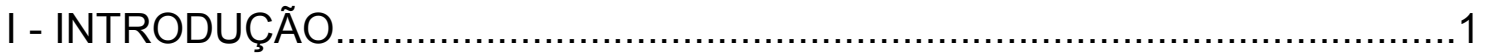

1. A susceptibilidade da criança à insuficiência respiratória ..................2

2. A ventilação pulmonar mecânica com pressão positiva.....................4

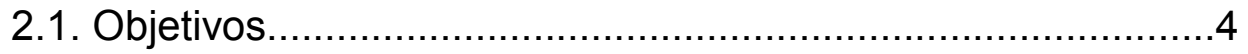

2.2. Indicações de ventilação pulmonar mecânica ........................5

2.3. Situações especiais......................................................

2.4. Trabalhos sobre prática da ventilação pulmonar mecânica na população pediátrica............................................................

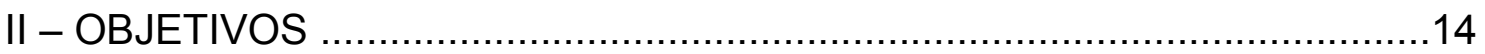

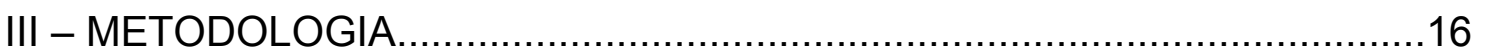


V - DISCUSSÃO 32

VI - CONCLUSÕES

VII - REFERÊNCIAS BIBLIOGRÁFICAS.

VIII - ANEXOS .52

Anexo 1 - Formulário de coleta de dados. 53

Anexo 2 - Aprovação do projeto junto à Comissão de Ética 55

Anexo 3- Certificado de apresentação de tema livre no VIII Congresso Panamericano e Ibérico de Medicina Crítica e Terapia Intensiva, 2007 .56 Anexo 4 - Artigo "How is mechanical ventilation employed in a pediatric intensive care unit in Brazil?", aceito para publicação na revista Clinics, 2009. 57 
$\underline{\text { I- Introdução }}$ 


\section{A susceptibilidade da criança à insuficiência respiratória}

As doenças respiratórias são altamente prevalentes na população pediátrica, especialmente nos países em desenvolvimento. O último boletim da Organização Mundial de Saúde (OMS) mostrava que, no Brasil, a pneumonia foi causa de $13 \%$ da mortalidade abaixo de cinco anos de idade, no ano de $2000[1]$

Na cidade de São Paulo, em particular, há uma tendência de aumento destas doenças. $49,6 \%$ das crianças menores de cinco anos apresentavam doença respiratória, com ou sem sibilância associada, nos anos de 95/96; sendo que uma década antes, este índice era de 29\% [2].

As crianças apresentam uma maior susceptibilidade anatômica à insuficiência respiratória. Embora as estruturas anatômicas dos sistemas condutores de ar já estejam presentes ao nascimento, seu tamanho absoluto e suas relações anatômicas variam muito entre o recém-nascido, o lactente, a criança maior e o adulto. Há um predomínio da respiração nasal até $04^{\circ}-6^{\circ}$ mês de vida. A parte mais estreita das vias aéreas superiores na criança é a cartilagem cricóide, na região subglótica e no adulto, são as cordas vocais. 0 menor calibre ou menor permeabilidade das pequenas vias aéreas leva a uma maior resistência ao fluxo de ar, com menor volume pulmonar. O suporte cartilaginoso das vias aéreas é mais frágil: aumenta em número até dois meses de idade, aumenta em área até o final da infância. A caixa torácica do lactente é mais complacente, distorce-se facilmente, com menos eficiência respiratória. Quanto mais jovem a criança, menor o número e o diâmetro dos alvéolos. Um recém-nascido a termo tem cerca de 20 milhões de sacos alveolares, com 2,8 $\mathrm{m}^{2}$ de área para troca gasosa. Uma criança de 8 anos tem cerca de 300 
milhões de alvéolos, com uma área alveolar para troca gasosa de $32 \mathrm{~m}^{2}$. Isto significa maior propensão ao colapso e menor reserva pulmonar, quanto menor a criança. A criança pequena ainda tem menor ventilação colateral, menor resistência à fadiga e maior propensão à apnéia por imaturidade dos centros respiratórios e menor resposta ventilatória à hipercapnia [3].

Há ainda uma maior susceptibilidade imunológica às infecções, pela imaturidade relativa do sistema imune da criança [4].

Além das doenças respiratórias, várias outras podem evoluir para a necessidade de suporte ventilatório, tais como sepse e choque séptico, doenças neuromusculares, pós-operatório, rebaixamento de sensório por traumatismo cranioencefálico, meningites, encefalopatia hepática. Assim sendo, a ventilação pulmonar mecânica (VPM) torna-se uma das principais indicações de internação em Unidade de Terapia Intensiva (UTI) Pediátrica [5]. 


\section{A Ventilação Pulmonar Mecânica com Pressão Positiva}

\subsection{Objetivos}

A ventilação pulmonar mecânica (VPM) tem sido amplamente empregada nas unidades de terapia intensiva, desde sua introdução na década de 50 , durante a epidemia de poliomielite. Trata-se de um dispositivo de suporte de vida, indicado nas situações em que o sistema respiratório deixa de satisfazer adequadamente as trocas gasosas e passa a comprometer a vida do paciente.

Quando a ventilação pulmonar mecânica é empregada, têm-se como objetivos [5]:

(a) Manter as trocas gasosas pulmonares: reverter a hipoxemia e aliviar a acidose respiratória aguda;

(b) Aliviar o desconforto respiratório: reduzir o gasto energético da respiração e reverter a fadiga dos músculos respiratórios;

(c) Controlar a ventilação: reduzir a pressão intracraniana e estabilizar a parede torácica;

(d) Alterar as relações de pressão/ volume: prevenir e reverter atelectasias, melhorar a complacência, evitar novas lesões;

(e) Permitir a cicatrização pulmonar e das vias aéreas. 


\subsection{Indicações de ventilação pulmonar mecânica}

"A VPM não está restrita às doenças pulmonares como insuficiência respiratória, estando também indicada em outras condições clínicas. O uso da VPM implica indicação criteriosa, experiência clínica e conhecimento adequado do equipamento utilizado" [5].

As principais indicações de ventilação mecânica são [6, 7]:

a) Rebaixamento do nível de consciência: por alteração metabólica (devido a evento metabólico primário, por exemplo, encefalopatia hepática), intoxicação secundária à ingestão voluntária ou acidental de drogas e substâncias ilegais, trauma de crânio, infecções do sistema nervoso central (meningites, encefalites), estado de mal epiléptico.

b) Doenças neuromusculares: disfunção do sistema nervoso periférico, massa muscular e/ou placa motora, gerando insuficiência respiratória.

c) Insuficiência respiratória crônica agudizada: exacerbação aguda de doença pulmonar crônica (devida a malformações respiratórias, displasia broncopulmonar, e fibrose cística, dentre outras patologias), estado de mal asmático e/ou exacerbação de broncoespasmo em paciente com história prévia de hiperreatividade brônquica.

d) Insuficiência respiratória aguda, por diversas causas:

Pós-operatório: pacientes que forem submetidos à cirurgia, que antes da mesma não faziam uso de ventilação mecânica e que após a mesma não foram extubados no período pós-operatório imediato.

Edema agudo de pulmão ou insuficiência cardíaca congestiva.

Broncoaspiração: pacientes nos quais se encontra conteúdo gástrico na via aérea ou no aspirado traqueal. 
Pneumonia: infiltrado radiológico alveolar associado a febre/ hipotermia e leucocitose/leucopenia.

Bronquiolite: taquipnéia, tosse, retrações torácicas, tempo expiratório prolongado, estertores pulmonares e hiperinsuflação pulmonar à radiografia de tórax, em pacientes menores de dois anos, com história sugestiva de infecção viral.

Sepse: síndrome de resposta inflamatória sistêmica frente a uma infecção suspeita ou comprovada [8].

Síndrome do desconforto respiratório agudo (SDRA): infiltrado alveolar bilateral à radiografia de tórax, presença de um evento agudo desencadeante, relação $\mathrm{PaO}_{2} / \mathrm{FiO}_{2}$ menor que 200, sem evidência de hipertensão capilar pulmonar

Trauma: pacientes com necessidade de ventilação mecânica devido a trauma abdominal, torácico ou múltiplo.

Parada cardíaca. 


\subsection{Situações especiais:}

\subsection{1- Lesão Pulmonar Aguda (LPA) e Síndrome do Desconforto Respiratório Agudo (SDRA).}

A lesão pulmonar aguda (LPA) caracteriza-se por processo inflamatório extenso que leva à quebra da barreira alvéolo-capilar, com desenvolvimento de edema intersticial e alveolar, diminuição da complacência pulmonar, desequilíbrio da relação ventilação-perfusão e hipoxemia refratária à administração de oxigênio. A lesão de pneumócitos tipo II, produtores de surfactante, e a inativação do surfactante pelo processo inflamatório também contribuem para o colabamento expiratório de unidades alveolares, com conseqüente redução da capacidade residual funcional. A SDRA é a forma clínica mais grave e o espectro final da LPA [9-11].

Os critérios diagnósticos, conforme a Conferência Americano-Européia de Consenso, de 1994, são: infiltrado alveolar bilateral à radiografia torácica, presença de um evento agudo desencadeante, relação $\mathrm{PaO}_{2} / \mathrm{FiO}_{2}$ menor que 300 para LPA e menor que 200 para SDRA, sem evidência de hipertensão capilar pulmonar (pressão pulmonar deve ser inferior a 18mmHg)[12].

Este processo pode ser causado por agressão pulmonar direta (SDRA primária), como ocorre na broncoaspiração, infecção pulmonar, afogamento e contusão pulmonar, ou pode ser provocado por agressão indireta (SDRA secundária), como na sepse, politraumatismo, transfusões maciças de hemoderivados $[13,14]$. 


\subsection{2- Lesão pulmonar associada ao ventilador}

Foram identificadas complicações relacionadas ao uso da VPM: infecções (pneumonia e sinusite associadas ao ventilador), toxicidade pelo oxigênio (retinopatia da prematuridade, displasia broncopulmonar) e, principalmente, lesões pulmonares induzidas pelas próprias estratégias ventilatórias [15].

VPM com altas pressões e altos volumes correntes modificam a permeabilidade endotelial e epitelial, induzem formação de edema pulmonar e alteram a permeabilidade microvascular pulmonar, gerando lesão alveolar grave, com hemorragia e formação de membranas hialinas: trata-se da lesão pulmonar induzida pelo ventilador ("Ventilator Induced Lung Injury - VILI”).

Os principais fatores implicados até o momento na patogênese da VILI são $[16,17]$ :

- Altos volumes e pressões pulmonares, por gerarem pressões transpulmonares elevadas e distensão alveolar excessiva (pelo volume corrente e pelo volume expiratório final): volutrauma / barotrauma.

- Colapso e reabertura alveolares repetidamente, devido principalmente a pressões positivas ao final da expiração (PEEPs) insuficientes: atelectrauma.

- Colapso das pequenas vias aéreas, principalmente nas regiões gravidade - dependentes, que ocorre antes do colapso alveolar, não é visto à tomografia computadorizada e já determina produção local e liberação sistêmica de mediadores inflamatórios: biotrauma. 


\subsection{Trabalhos sobre prática da VPM na população pediátrica}

Pacientes adultos habitualmente são ventilados a volume-controlado [18, 19], entretanto, não há uniformidade no que seria o modo "convencional" de se ventilar crianças. Em nosso meio, há grande prática de ventilação a pressãocontrolada, para outros, o tradicional seria ventilar a volume-controlado $[20,21]$. Apresentamos, a seguir, os principais trabalhos publicados na última década, sobre o emprego da VPM na população pediátrica, com suas respectivas datas de publicação.

\subsection{1 - Estudo Multicêntrico PALISI, 2002 [22]}

O "PALISI - Pediatric Acute Lung Injury and Sepsis Investigators Network" é um consórcio de pesquisadores que estudam tratamentos para lesão pulmonar aguda e sepse em crianças. Como parte de um ensaio clínico para avaliação de técnicas de desmame ventilatório, foram coletados dados de pacientes durante 6 meses (15/10/00 a 15/04/01), em 9 UTIs na América do Norte: 8 nos EUA e 1 no Canadá. Dos 6403 pacientes pediátricos admitidos no período, $1096(17,1 \%)$ necessitaram de ventilação mecânica por, pelo menos, 24 horas. Como o objetivo do trabalho foi avaliar a elegibilidade de pacientes a ensaios clínicos, muitos pacientes foram excluídos. 395 crianças (36\%) não apresentaram critérios de exclusão e $76,7 \%$ delas foram avaliadas diariamente ( $n=303)$. As demais não foram avaliadas por falta de consentimento.

A duração média da ventilação mecânica foi de 9,5 dias (mediana de 8 dias). A principal causa de ventilação mecânica foi bronquiolite, seguida por pneumonia. 
Apenas $7,6 \%$ dos pacientes estudados apresentaram diagnóstico de SDRA à entrada do estudo. Entretanto, $52 \%$ das crianças apresentaram $\mathrm{PaO}_{2} / \mathrm{FiO}_{2}<200$ por mais de um dia, sendo possível que a SDRA tenha sido subdiagnosticada ou que estas crianças tenham desenvolvido SDRA ao longo da internação. Os autores relatam uma mortalidade de SDRA de apenas 4,3\%, que pode ter sido ainda menor, dado o subdiagnóstico. Entretanto, esta mortalidade tão baixa é atribuída à bronquiolite ter sido o principal diagnóstico etiológico.

\subsection{2- Estudo Multicêntrico IGMVC, 2004 [6]}

Em 2004, Farias e colaboradores publicaram os resultados do "International Group of Mechanical Ventilation in Children - IGMVC", uma coorte prospectiva de crianças que foram submetidas à ventilação mecânica por mais de doze horas. O trabalho foi realizado por dois meses, em 36 unidades de terapia intensiva pediátrica e 1893 crianças foram admitidas entre $1^{\circ}$ de abril e 31 de maio de 1999 . 35\% foram submetidas à ventilação mecânica (659 crianças): 39\% em SIMV com ou sem pressão de suporte, 25\% em pressão-controlada e $26 \%$ foram ventiladas a volume, em ventilação assistido/controlada. 53\% receberam mais de uma modalidade ventilatória. 0 tempo mediano de VPM foi de quatro dias. $72 \%$ dos pacientes apresentaram insuficiência respiratória aguda como causa de VPM. Apenas $2 \%$ receberam diagnóstico de SDRA. A mortalidade geral da UTI no período foi de $15 \%$, mas nas crianças com SDRA foi de $50 \%$.

Este estudo apresentou pontos extremamente importantes: foi um trabalho pioneiro, multicêntrico, que estudou um grande número de pacientes, 
adequado para dar força estatística ao estudo, mas o estudo foi realizado em 1999, antes da incorporação à prática clínica da estratégia de ventilação protetora (menores volumes correntes), iniciada por Amato em 1998 e reafirmada pela ARDS Network em 2000 [23, 24].

\subsection{3- Estudo Multicêntrico Espanhol, 2004 [25].}

Estudo observacional, realizado através de questionário escrito enviado às 46 UTIPs espanholas, no qual deveriam ser informados dados referentes aos pacientes submetidos à ventilação mecânica no dia 19 de fevereiro de 2002. 31 UTIPs responderam, sendo que somente 27 tinham pacientes ventilados no dia do estudo. A prevalência de ventilação mecânica foi de 86 pacientes (45,5\%), com uma idade média de 36 e mediana de 8 meses. $60 \%$ eram do gênero masculino. A principal indicação de VPM foi insuficiência respiratória aguda (46,5\%), seguida de coma (11,6\%). 23,2\% dos pacientes eram ventilados por traqueostomia. Em $30 \%$ dos casos, a duração da VPM foi maior do que um mês. $46,5 \%$ dos pacientes foram ventilados em modalidades a pressão, $32,5 \%$ a volume e apenas $2,3 \%$ foram submetidos à ventilação de alta freqüência.

\subsection{4- Estudo Indiano, 2005 [21]}

Trabalho retrospectivo de uma UTI Pediátrica em Nova Delhi, Índia, cujo objetivo primário foi comparar ventilação controlada a volume com ventilação pressão-regulada e volume-controlada (PRVC). 189 pacientes foram admitidos entre julho e dezembro de 2000 e 44 pacientes foram submetidos à VPM. 16 pacientes foram excluídos: 11 por receberem ventilação a pressão, dois por serem recém-nascidos e 3 por terem sido ventilados por menos de 24 horas. 
Dos 28 pacientes estudados, 14 receberam ventilação a volume e 14 receberam PRVC. Não houve diferença no tempo de ventilação, possivelmente devido ao pequeno tamanho da amostra.

\subsection{5- Estudo Multicêntrico na República Checa, 2005 [26]}

Estudo observacional prospectivo realizado em sete UTIs pediátricas de hospitais terciários na República Checa. Dos pacientes admitidos entre 01/02/2002 e 30/04/2002, cento e quarenta e quatro (23\%) receberam ventilação mecânica. A idade média dos pacientes ventilados foi de 70 meses e o peso médio, de $23 \mathrm{~kg}$. O tempo médio de VPM foi de 117 horas. $66 \%$ dos pacientes tinham insuficiência respiratória aguda por uma causa extrapulmonar. Apenas 19\% apresentavam doença crônica e somente 0,7\% encontravam-se imunossuprimidos. As modalidades ventilatórias mais utilizadas foram PRVC e pressão positiva bifásica. Ventilação não-convencional foi utilizada em $13 \%$ dos pacientes. A mortalidade foi de $3,5 \%$.

\subsection{6- Estudo Turco, 2006 [27]}

Foi realizada análise retrospectiva de prontuários dos 407 pacientes admitidos em uma UTIP em Ankara, Turquia, de janeiro de 2002 a maio de 2005. 91 pacientes $(22,3 \%)$ foram submetidos à ventilação mecânica. A idade mediana foi de 8 meses. A duração da ventilação mecânica foi de 18,8 \pm 14,1 dias. A principal indicação de ventilação mecânica foi insuficiência respiratória aguda (64,8\%). Quatro crianças foram traqueostomizadas. Embora a mortalidade geral da UTIP tenha sido $12,2 \%$, a mortalidade dos pacientes ventilados foi de $58,3 \%$. 
Não se conhece 0 quanto a prática apresentada em estudos internacionais é semelhante à nossa. Embora trinta das trinta e seis UTIPs $(83,3 \%)$ do estudo IGMVC, por exemplo, estivessem na Espanha, América Central e do Sul, não havia nenhuma no Brasil [6].

Outro aspecto importante é a diferença de mortalidade entre os estudos, principalmente nos pacientes com lesão pulmonar aguda. No trabalho de Randolph, embora a maioria dos pacientes tivesse doença de baixa mortalidade (bronquiolite), $52,2 \%$ dos pacientes com SDRA receberam ventilação oscilatória de alta freqüência e a mortalidade foi de apenas 4,3\%[22]. No trabalho de Farias, quando a ventilação de alta freqüência ainda não era difundida na prática clínica e somente 1,5\% recebeu tal modalidade, a mortalidade dos pacientes com SDRA chegou a 50\%[6].

A ventilação de alta freqüência ainda não é usual em nossa prática clínica. Entretanto, trabalhos experimentais e estudos clínicos vêm mostrando que não parece haver diferença entre a ventilação de alta freqüência e a ventilação com estratégia protetora adequada, quanto à morbimortalidade [28, 29].

Para que possa haver máxima adequação do tratamento às melhores evidências científicas disponíveis, torna-se necessário conhecer a prática de ventilação mecânica a qual são submetidos os pacientes assistidos na UTI Pediátrica do Instituto da Criança. 
II-Objetivos 
Objetivo geral:

- Descrever a prática da ventilação mecânica em crianças admitidas na UTI pediátrica de um hospital de nível terciário, na cidade de São Paulo, Brasil.

Objetivos específicos:

- Explorar fatores associados à ventilação mecânica que constituam risco para maior tempo de VPM e mortalidade em até 28 dias da admissão à UTIP.

- Avaliar se as estratégias de ventilação protetora são utilizadas em crianças com lesão pulmonar aguda. 
$\underline{\text { III-Metodologia }}$ 
O desenho do estudo foi o de coorte prospectiva de todos os pacientes admitidos na UTIP do Instituto da Criança do HC-FMUSP, submetidos à VM por 24 horas ou mais, durante 01/10/2005 e 31/03/2006.

\section{Critérios de inclusão}

Foram utilizados como critérios de inclusão no estudo:

(1) admissões consecutivas na UTI Pediátrica do Instituto da Criança entre 01/10/2005 e 31/03/2006,

(2) uso de ventilação mecânica por período de 24 horas ou superior.

\section{Critérios de exclusão}

Os critérios de exclusão se basearam na impossibilidade de descrição das variáveis (ausência de coleta de gasometria ou pacientes ventilados em outros aparelhos, sem medida de volume corrente).

Também foram excluídos da análise os pacientes com cardiopatia e/ou hipertensão pulmonar prévias, com comprometimento da oxigenação; aqueles previamente dependentes de ventilação mecânica, como também os ventilados em outra instituição por mais de 24 horas, pela possibilidade de alterações pulmonares decorrentes das estratégias ventilatórias utilizadas.

Dois pacientes receberam ventilação de alta freqüência, após um período de ventilação a pressão. Nestes, foram analisados somente os dados da ventilação a pressão.

\section{Variáveis e desfechos}

As variáveis descritas foram: idade, peso, gênero, indicação de ventilação mecânica, acesso à via aérea, modos ventilatórios, parâmetros do respirador (pressões, freqüência respiratória, volume corrente, $\mathrm{FiO}_{2}$ ), dados gasométricos, disfunção de órgãos e sistemas.

Foi utilizada a definição de disfunção de órgãos dada pela Conferência Internacional de Consenso em Sepse Pediátrica [8].

Os desfechos analisados foram dias de ventilação pulmonar mecânica e mortalidade em 28 dias da admissão à UTIP. 


\section{Coleta dos dados}

Os dados foram prospectivamente coletados em formulários baseados no trabalho original de Farias e colaboradores, adaptados para os objetivos do trabalho (anexo 1)[6].

Somente duas pesquisadoras (DCBS, AROS) efetuaram a coleta dos dados. Para complementar a avaliação dos desfechos, os prontuários foram revistos após a alta hospitalar de cada paciente.

Em relação ao dado de volume corrente, foi utilizado o volume corrente expiratório medido pelo aparelho, para diminuir a influência de escape de ar pelas cânulas, já que nem todas possuíam balão insuflável ("cuff").

\section{Aspectos éticos}

O protocolo deste estudo foi revisto e aprovado pela Comissão de Ética em Pesquisa da instituição, previamente ao início da coleta de dados (anexo 2).

Para que o trabalho representasse a prática da VPM em uso, a equipe de assistência não estava ciente do mesmo, somente o coordenador da UTIP.

\section{Análise estatística}

A análise estatística foi realizada através do software StatView versão 5.01 (SAS Institute). Dados são mostrados em medianas (com intervalos interquartilares) e proporções (com intervalos de 95\% de confiança). As variáveis de distribuição não-normal foram analisadas pelo teste $U$ de MannWhitney. Foi considerado significativo $p<0,05$. Os fatores de risco são apresentados com suas respectivas razões de chance ("odds ratio").

Em relação ao subgrupo com LPA, foi feita uma análise complementar através do software Stata. Foi realizada uma análise univariada e todos os parâmetros com $p<0,1$ foram selecionados para análise multivariada (regressão logística). Nesta última, também foi considerado significativo $\mathrm{p}<0,05$. 


\section{O Instituto da Criança e sua UTIP}

O Instituto da Criança é um hospital de referência de nível terciário, conforme a legislação do Sistema Único de Saúde - SUS, em vigor [30]. É um hospital pediátrico de 135 leitos, sendo 13 na UTIP, à época do estudo.

A equipe médica da UTIP era composta por dois intensivistas pediátricos (um diarista e um plantonista) e seis residentes durante o dia, além de um intensivista preceptor. Durante o plantão, havia sempre um médico intensivista pediátrico, prestando assistência direta e supervisionando a atividade dos residentes.

\section{O respirador}

Foi utilizado respirador artificial já existente na própria UTIP, no qual estavam disponíveis, além da medida de volume corrente expirado, os modos ventilatórios a volume, a pressão e a pressão com volume alvo (VTPC), nas modalidades assistido-controlado $(\mathrm{A} / \mathrm{C})$, mandatório intermitente sincronizado com pressão de suporte (SIMV + PS) e espontâneo (Newport E500®, Newport NMI, USA). 
IV-Resultados 
Foram admitidos 241 pacientes entre 01/10/2005 e 31/03/2006. Oitenta e seis foram submetidos à ventilação mecânica por 24 horas ou mais. Trinta e sete preencheram critérios de exclusão (quadro 1). Analisaram-se dados de 49 pacientes, de mediana de idade 32 meses (P25; P75: 1.87; 62.12) e de peso 13 kg (P25; P75: 7.77; 18.22) (tabela 1). 61,23\% dos pacientes eram do gênero masculino. Não houve perda de dados de pacientes.

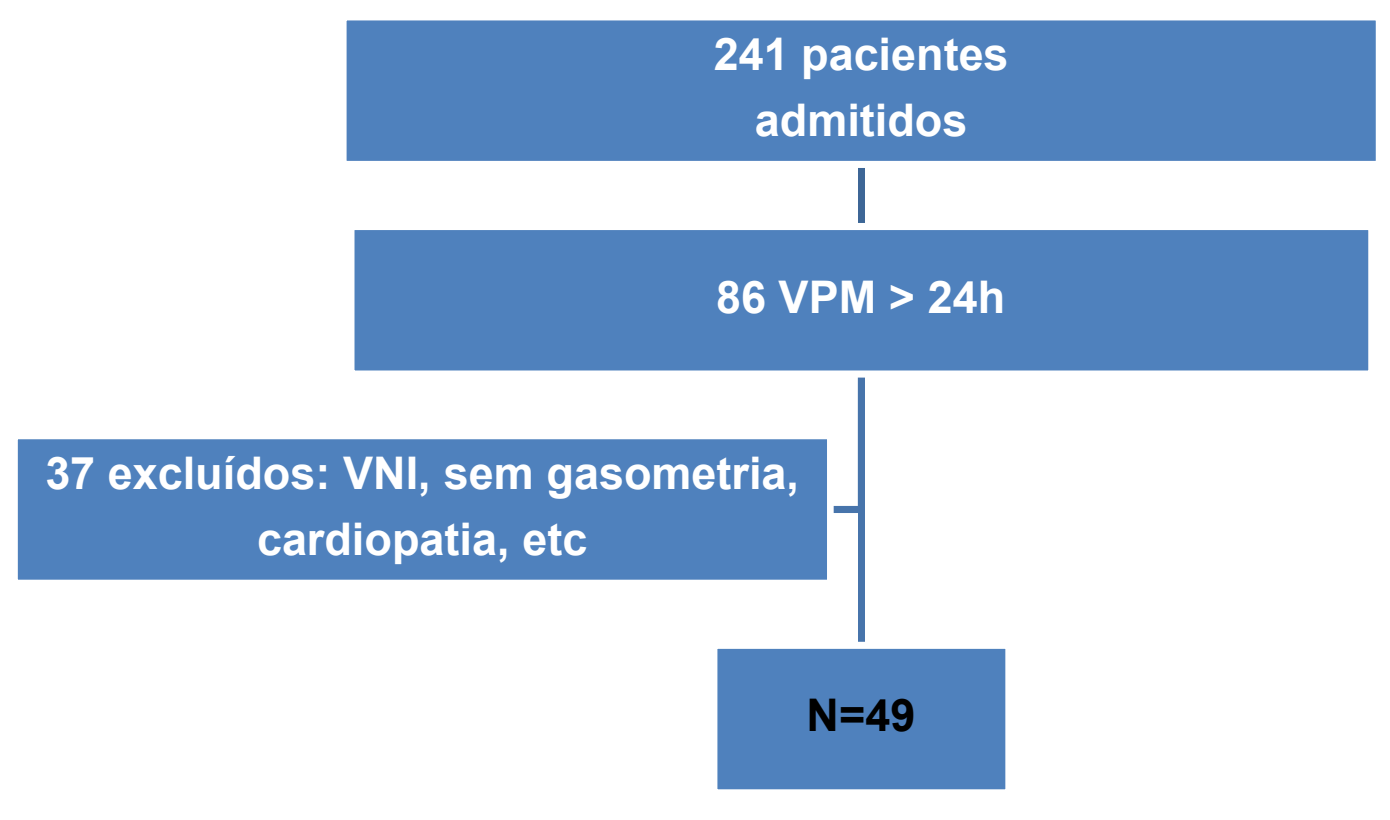

Quadro 1 - Identificação dos pacientes incluídos no estudo.

Apenas 4 dos 49 pacientes (8,16\%) não apresentavam doença crônica. As doenças crônicas mais prevalentes foram: respiratória $(n=13 ; 26,53 \%)$, neurológica $(n=9 ; 18,37 \%)$, hepática $(n=6 ; 12,24 \%)$ e oncológica $(n=7$; $14,28 \%)$. 


\section{Indicação da VPM}

A principal indicação da ventilação pulmonar mecânica foi insuficiência respiratória aguda $(63,2 \%)$, seguida pela exacerbação aguda de doença pulmonar crônica (18,3\%) e pelo rebaixamento do nível de consciência (16,3\%). Dos pacientes com insuficiência respiratória aguda, 17 se apresentavam com sepse grave / choque séptico.

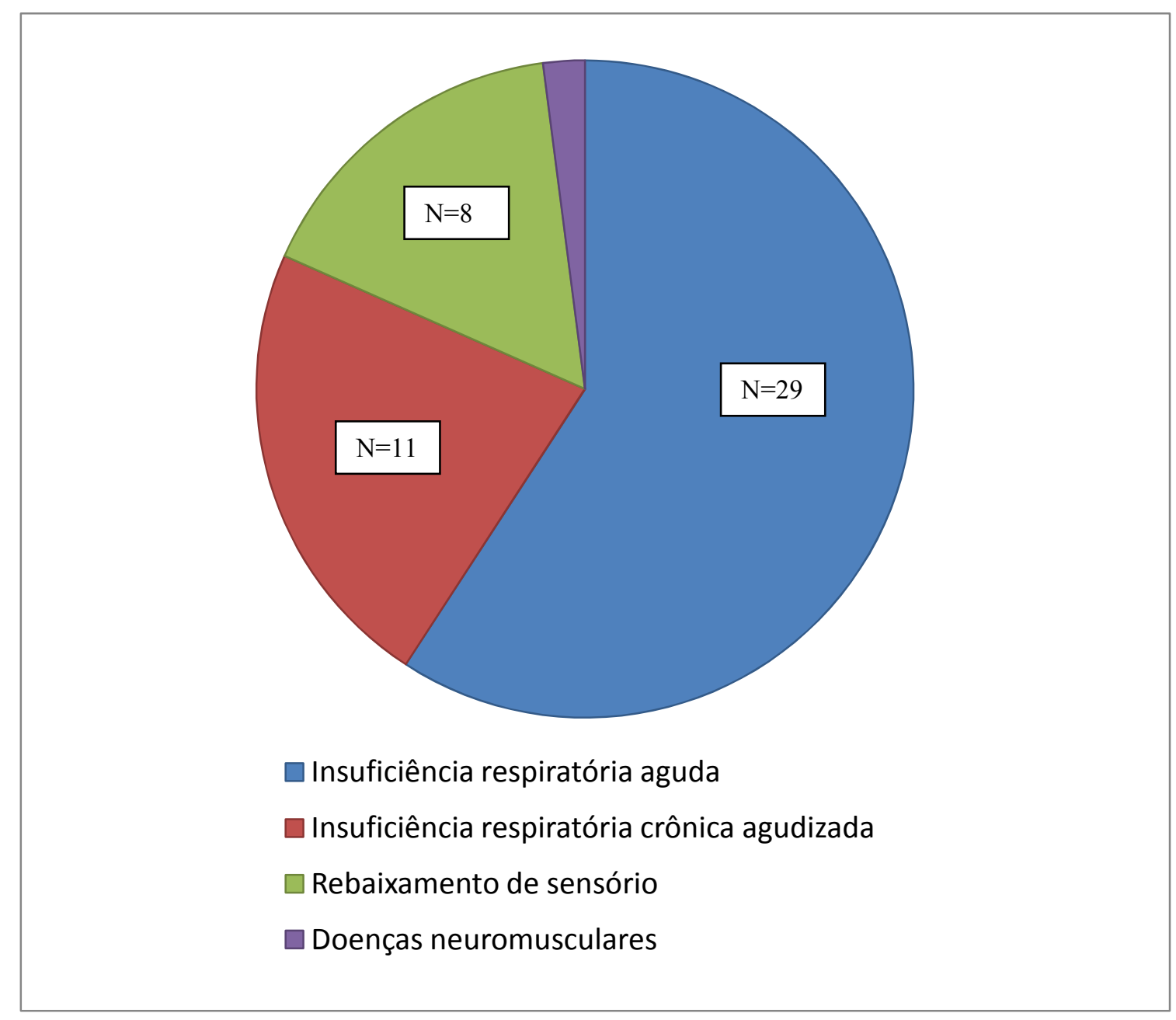

Gráfico 1 - Indicação da ventilação pulmonar mecânica 


\section{Acesso à via aérea}

A canulização da via aérea se deu preferencialmente por via orotraqueal.

Não houve intubação nasotraqueal. Cinco pacientes eram previamente traqueostomizados, outros 3 foram traqueostomizados após 3 semanas de VPM.

\section{Modalidades e parâmetros ventilatórios}

Nenhuma criança foi ventilada a volume: 48 foram ventiladas inicialmente à pressão e uma à pressão com volume alvo.

Quarenta e sete por cento dos pacientes foram submetidos a apenas uma modalidade ventilatória durante sua internação (A/C ou SIMV + PS) e 53\% a mais de uma.

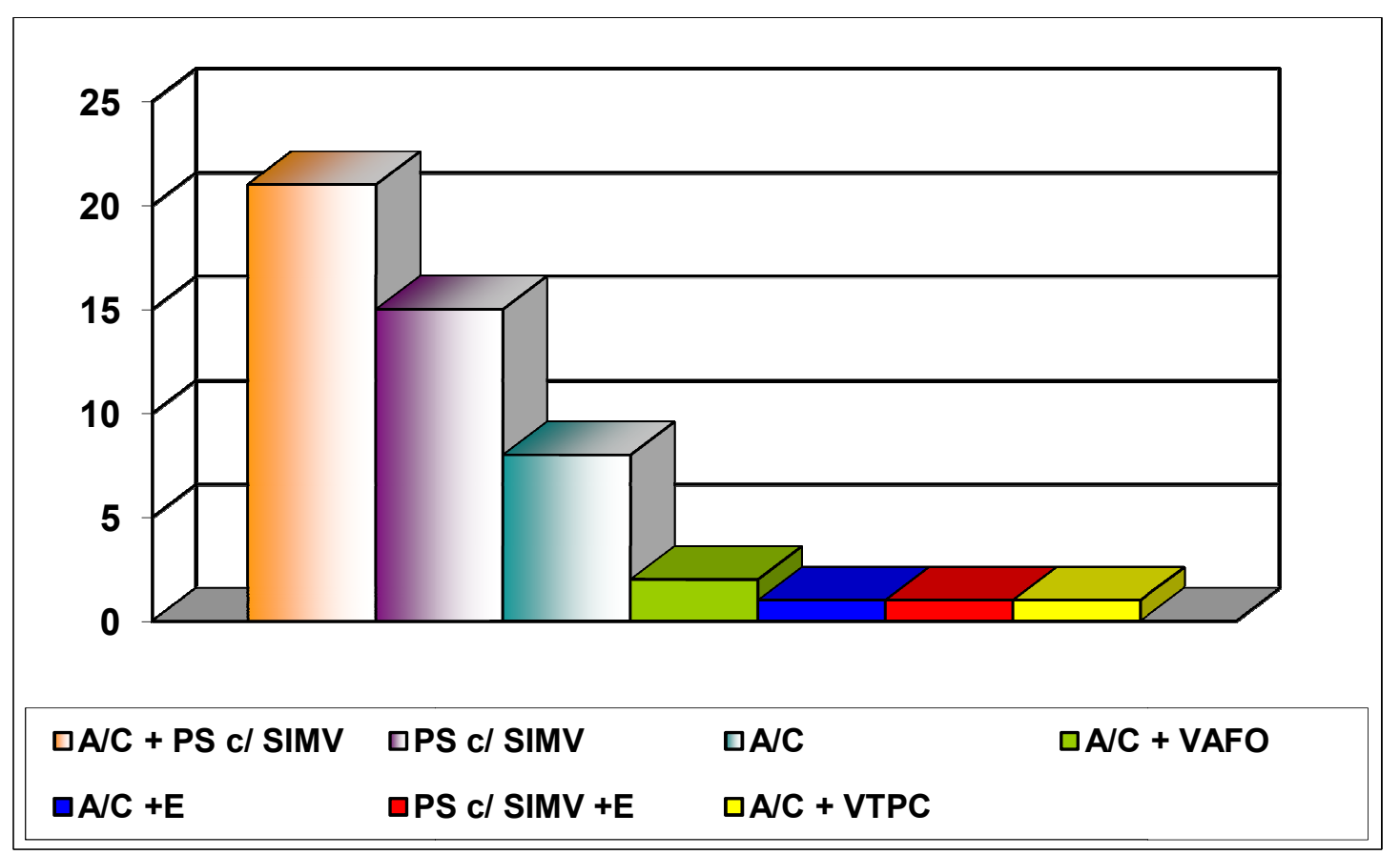

Gráfico 2 - Modalidades ventilatórias utilizadas na população estudada. 
Tabela 1- Parâmetros ventilatórios, dados gasométricos e disfunção de órgãos

\begin{tabular}{|c|c|c|}
\hline Variável & Mediana & P25 - P75 \\
\hline Dias de VPM & 6.50 & $2.50-10.50$ \\
\hline Volume corrente inicial (ml/kg) & 10 & $9-11$ \\
\hline $\begin{array}{l}\text { Volume corrente com } 48 \mathrm{~h} \text { de } \text { VPM } \\
(\mathrm{ml} / \mathrm{kg})\end{array}$ & 9.5 & $8.19-10.81$ \\
\hline PEEP inicial & 8 & $6-10$ \\
\hline PEEP com $48 \mathrm{~h}$ de VPM & 8 & $6-10$ \\
\hline Freqüência respiratória $(\mathrm{FR})$ inicial & 20 & $17.5-22.5$ \\
\hline FR com 48 h de VPM & 20 & $15.5-24.5$ \\
\hline Pressão inspiratória inicial & 24 & $20.37-27.62$ \\
\hline Pressão inspiratória com 48h de VPM & 24 & $19.25-28.75$ \\
\hline $\mathrm{pH}$ inicial & 7.36 & $7.27-7.45$ \\
\hline $\mathrm{pH}$ com $48 \mathrm{~h}$ de VPM & 7.38 & $7.32-7.49$ \\
\hline $\mathrm{PaO}_{2} / \mathrm{FiO}_{2}$ inicial & 245.1 & $159.11-331.08$ \\
\hline $\mathrm{PaO}_{2} / \mathrm{FiO}_{2}$ com $48 \mathrm{~h}$ de VPM & 237.0 & $146.76-327.23$ \\
\hline Índice de oxigenação (IO) inicial & 6 & $3.51-8.49$ \\
\hline IO com 48 h de VPM & 5.8 & $3.81-7.79$ \\
\hline PELOD & 11 & $6-16$ \\
\hline Disfunção orgânica inicial & 2 & $1.5-2.5$ \\
\hline Disfunção orgânica com 48h de VPM & 2 & $0.5-3.5$ \\
\hline
\end{tabular}

VPM: Ventilação Pulmonar Mecânica; PEEP: Pressão Positiva ao Final da Expiração; FR: Freqüência Respiratória; PaO2: Pressão parcial arterial de oxigênio; FiO2: Fração Inspiratória de oxigênio; IO: Índice de Oxigenação; PELOD: "Pediatric Logistic Organ Dysfunction".

\section{Volume Corrente}

Foram utilizados volumes correntes superiores a $10 \mathrm{ml} / \mathrm{kg}$ em 13 pacientes após 48h sob VPM. Apesar disso, não foi observado nenhum pneumotórax nos pacientes estudados. 


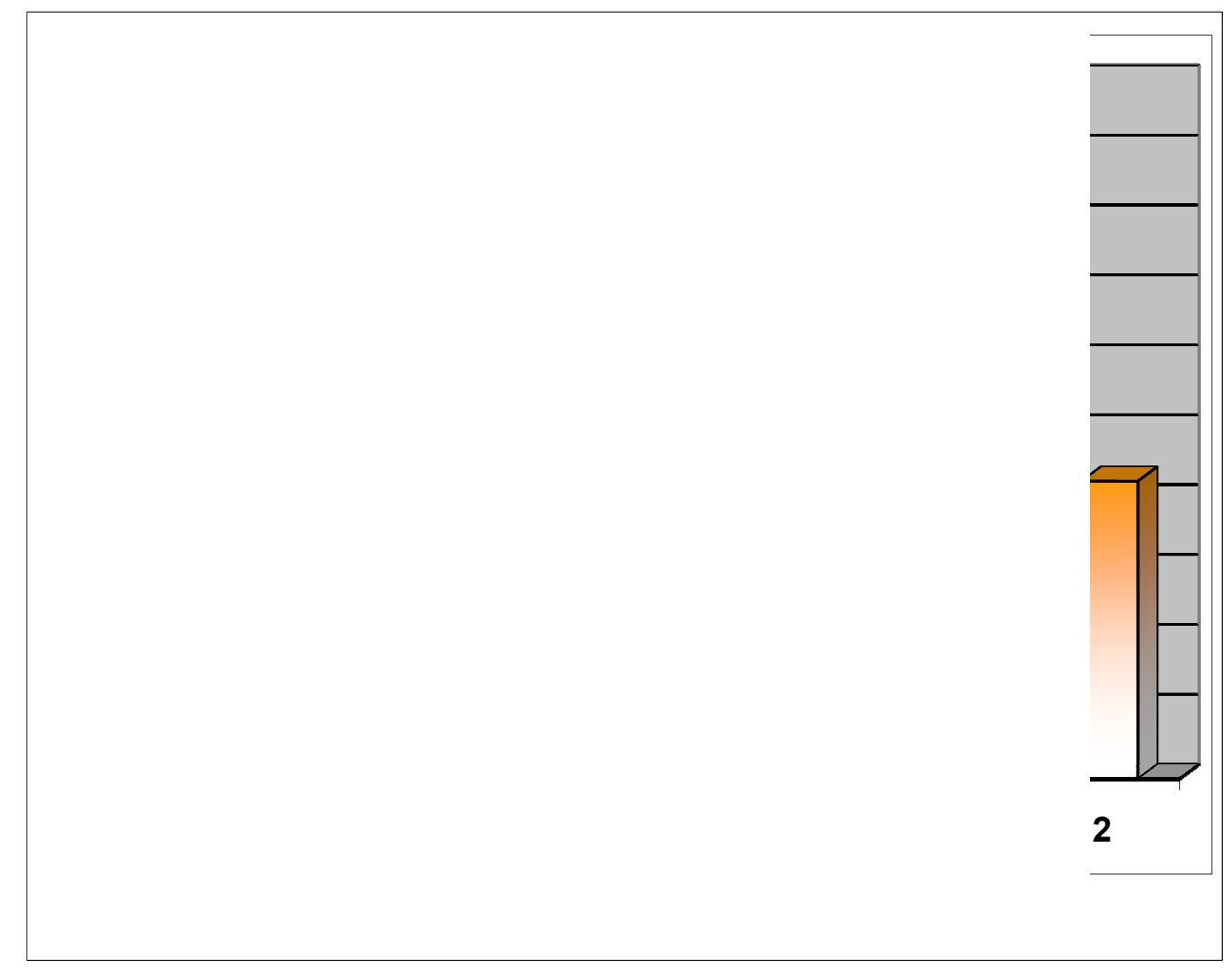

Gráfico 3 - Volumes correntes utilizados nos pacientes estudados, ao início e após 48 horas de ventilação mecânica.

\section{Pressão Inspiratória}

Embora nenhum paciente tenha sido ventilado inicialmente com pressão inspiratória superior a $30 \mathrm{cmH}_{2} \mathrm{O}$, nove pacientes o foram após 48 horas (tabela 2). 


\begin{tabular}{|l|l|l|}
\hline Pressão Inspiratória & Início VPM & 48 horas de VPM \\
\hline$\leq 25 \mathrm{cmH}_{2} \mathrm{O}$ & 30 & 29 \\
\hline $26-30 \mathrm{cmH}_{2} \mathrm{O}$ & 19 & 11 \\
\hline $31-35 \mathrm{cmH}_{2} \mathrm{O}$ & 0 & 6 \\
\hline $36-40 \mathrm{cmH}_{2} \mathrm{O}$ & 0 & 1 \\
\hline$\geq 41 \mathrm{cmH}_{2} \mathrm{O}$ & 0 & 2 \\
\hline
\end{tabular}

Tabela 2 - Pressões inspiratórias utilizadas nos pacientes estudados, ao início e após 48 horas de VPM.

\section{Pressão Positiva ao Final da Expiração (PEEP)}

A mediana da PEEP foi de 8 (tanto ao início, quanto com 48h VPM), sendo a mínima utilizada de $5 \mathrm{cmH}_{2} \mathrm{O}$ e a máxima de 18. Dezessete pacientes receberam PEEP superior a $8 \mathrm{cmH} 2 \mathrm{O}$ durante o período em que estiveram ventilados.

\section{Pressão Média das Vias Aéreas}

A pressão média de vias aéreas (PmVA), com 48h de VPM, apresentou mediana de 12,6 ( $\left.P_{25} 11,3, P_{75} 17\right)$.

\section{Relação $\mathrm{PaO}_{2} / \mathrm{FiO}_{2}$}

Quarenta e um dentre os 49 pacientes apresentaram relação $\mathrm{PaO}_{2} / \mathrm{FiO}_{2}<300$, sendo o diagnóstico gasométrico de Lesão Pulmonar Aguda confirmado com critérios clínicos e radiológicos.

Destes, 25 apresentaram $\mathrm{PaO}_{2} / \mathrm{FiO}_{2}<200$ e demais critérios compatíveis com 
síndrome do desconforto respiratório agudo (SDRA). Dois foram submetidos a manobras de recrutamento alveolar e ventilados com alta freqüência após um dia de ventilação mecânica a pressão. Dois pacientes receberam insuflação traqueal de gases (oxigênio), sendo o fluxo de oxigênio utilizado de 3 litros por minuto. Não houve emprego de surfactante exógeno ou óxido nítrico inalatório, por não estarem disponíveis na unidade. A posição prona foi empregada aleatoriamente, sem controle do tempo em que o paciente permaneceu em decúbito ventral.

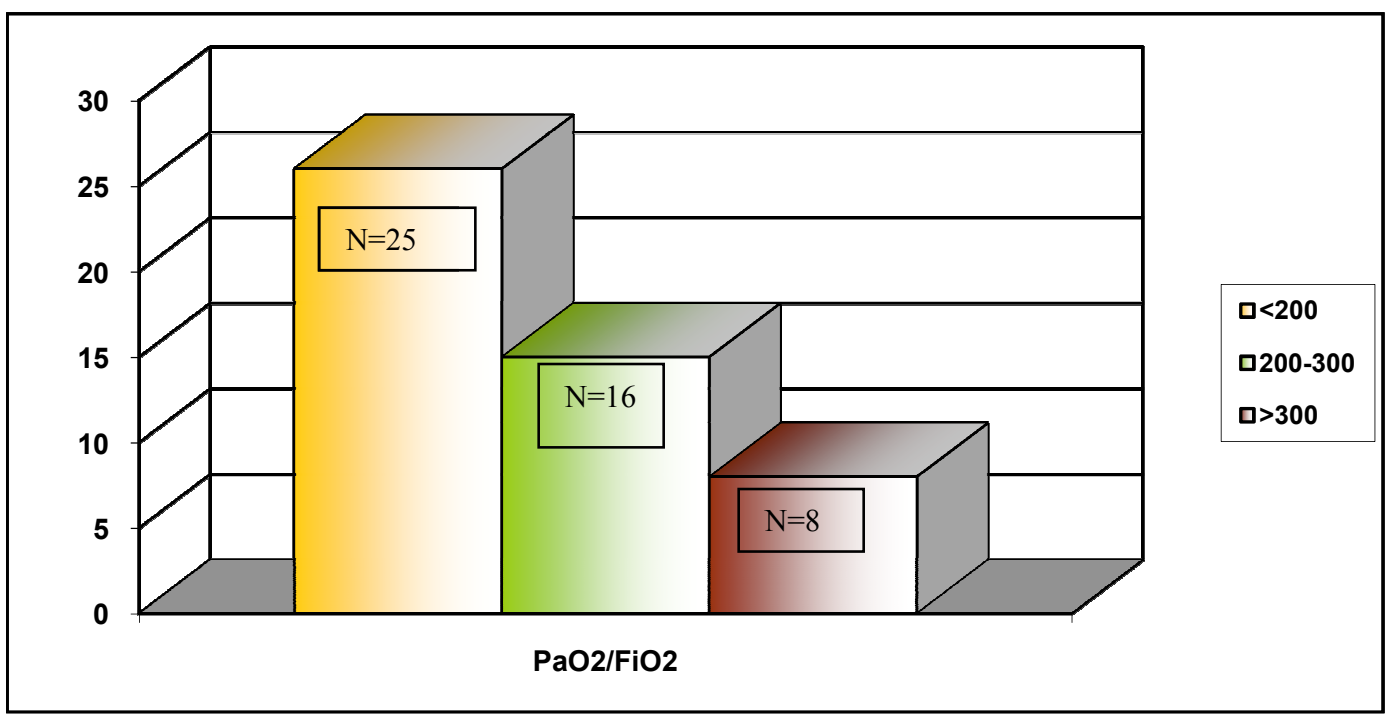

Gráfico 4 - Distribuição dos pacientes estudados quanto à relação $\mathrm{PaO}_{2} / \mathrm{FiO}_{2}$. 


\section{$\mathrm{PaCO}_{2}$}

A maioria dos pacientes incluídos neste estudo apresentou normo ou hipocapnia.

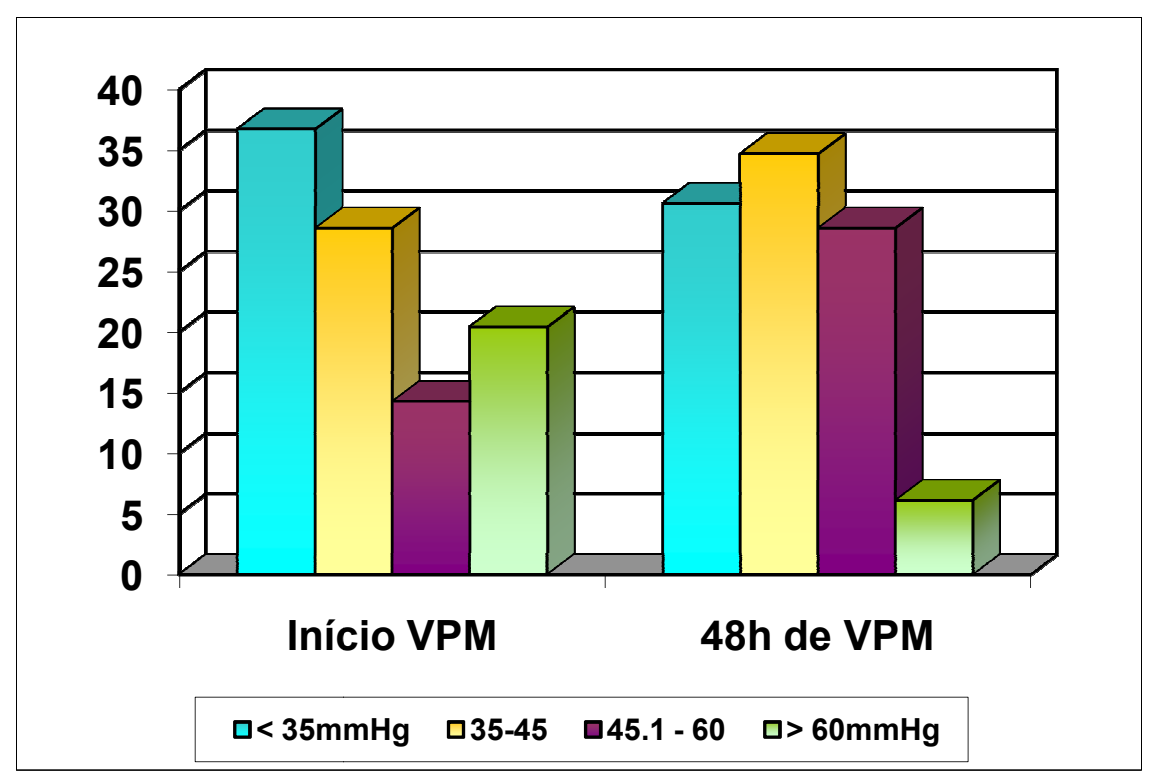

Gráfico 5 - Distribuição dos pacientes estudados quanto à pressão arterial de gás carbônico $\left(\mathrm{PaCO}_{2}\right)$, ao início e com 48 horas de ventilação mecânica.

\section{Índice de Oxigenação}

Seis pacientes apresentaram índices de oxigenação $(\mathrm{IO}=\mathrm{PmVA} \times \mathrm{FiO} 2 \times 100 /$ PaO2) superiores a 14 (12,2\%), com mortalidade de 100\% (5 na UTI e o outro em 28 dias da admissão).

\section{Dias de VPM}

A mediana de dias sob ventilação mecânica foi de $6,5\left(P_{25} 2,5, P_{75} 10,5\right)$. 


\section{Disfunção de órgãos e mortalidade em 28 dias.}

Sessenta e sete por cento dos pacientes apresentaram duas ou mais disfunções orgânicas (n=33).

O índice prognóstico "PELOD" utilizado obteve uma pontuação mediana de 11 , o que corresponderia a uma mortalidade teórica de 1,3\%.

Entretanto, após 28 dias de admissão à UTI, 17 pacientes haviam evoluído a óbito $(34,69 \%)$.

\section{Fatores de risco}

Pressão inspiratória, $\mathrm{pH}, \mathrm{PaO}_{2} / \mathrm{FiO}_{2}$ e índice de oxigenação ao início da VPM, além do índice de oxigenação com 48 horas de VPM, foram identificados como fatores de risco para mortalidade (tabela 3). Suas razões de chance ("odds ratio") são mostradas na tabela 4.

Tabela 3 - Fatores de risco para mortalidade em 28 dias

\begin{tabular}{|l|l|}
\hline Fator de risco & P (para mortalidade) \\
\hline Pressão inspiratória inicial & $p 0.005\left(^{*}\right)$ \\
\hline $\mathrm{pH}$ inicial & $p 0.049\left(^{*}\right)$ \\
\hline $\mathrm{PaO}_{2} / \mathrm{FiO}_{2}$ inicial & $p 0.016\left(^{*}\right)$ \\
\hline $\mathrm{IO}$ inicial & $p 0.007\left(^{*}\right)$ \\
\hline IO com 48 h VPM & $p 0.011\left(^{*}\right)$ \\
\hline PEEP inicial & $p 0.060$ \\
\hline Volume corrente inicial & $p 0.900$ \\
\hline
\end{tabular}

${ }^{*} \mathrm{p}<0.05$, teste de Mann-Whitney. 
Tabela 4 - Fatores de risco para mortalidade e duração de VPM superior a 7 dias, com suas respectivas razões de chance ("odds ratio").

\begin{tabular}{|c|c|c|}
\hline Fator de risco & $\begin{array}{l}\text { OR para Mortalidade } \\
(95 \% \text { IC) }\end{array}$ & $\begin{array}{l}\text { OR para duração VPM } \\
>7 \text { dias }(95 \% \text { IC) }\end{array}$ \\
\hline $\begin{array}{l}\text { Pressão inspiratória inicial } \\
>25 \mathrm{cmH}_{2} \mathrm{O}\end{array}$ & $5.5(1.53-19.71)$ & $5.96(1.69-21.03)$ \\
\hline $\begin{array}{l}\text { Volume corrente inicial } \\
>8 \mathrm{ml} / \mathrm{kg}\end{array}$ & $1.9(0.30-3.94)$ & $1.77(0.50-6.31)$ \\
\hline $\mathrm{pH}$ inicial $<7,20$ & $6(1.26-28.55)$ & $1.88(0.44-8.07)$ \\
\hline $\mathrm{PaO}_{2} / \mathrm{FiO}_{2}$ inicial $<200$ & $2.14(0.65-7.13)$ & $2.15(0.65-7.13)$ \\
\hline $\mathrm{PaO}_{2} / \mathrm{FiO}_{2}$ inicial $<100$ & $10.67(1.08-105.29)$ & $6.35(0.65-61.73)$ \\
\hline IO inicial $>10$ & $6.77(1.46-31.30)$ & $4.16(0.93-18.72)$ \\
\hline IO inicial $>14$ & $16.91(1.83-156.62)$ & ------ \\
\hline IO com $48 \mathrm{~h} V P M>10$ & $4.83(0.97-23.98)$ & ------ \\
\hline IO com 48 h VPM $>14$ & $11.27(1.13-112.07)$ & $4.06(0.7-23.47)$ \\
\hline
\end{tabular}


A análise univariada (regressão simples) demonstrou correlação entre dias de VPM e pressão inspiratória inicial ( $\left.p 0.015 ; R^{2} 0.130\right)$ e entre dias de VPM e relação $\mathrm{PaO}_{2} / \mathrm{FiO}_{2}$ inicial ( $\mathrm{p} 0.040 ; \mathrm{R}^{2}$ 0.111). Quando suas razões de chance foram calculadas, apenas a pressão inspiratória inicial foi preditiva de VPM por tempo superior a 7 dias, com uma razão de chance (OR) de 5,96 vezes (IC 95\% $1.69-21.03)$ (tabela 4).

No subgrupo de pacientes com lesão pulmonar aguda, também foi realizada análise univariada. Todas as variáveis com $p<0,1$ foram escolhidas para análise multivariada: pressão inspiratória e índice de oxigenação iniciais, pressão inspiratória, $\mathrm{PEEP}, \mathrm{pH}$, relação $\mathrm{PaO}_{2} / \mathrm{FiO}_{2}$ e índice de oxigenação em 48h de VPM. Foram identificados como fatores de risco para mortalidade: pressão inspiratória inicial, pH, índice de oxigenação e relação $\mathrm{PaO}_{2} / \mathrm{FiO}_{2}$ em 48h de VPM. Em relação ao tempo de VPM, somente houve correlação com a relação $\mathrm{PaO}_{2} / \mathrm{FiO}_{2}$ em 48h de VPM (p 0,039; $\left.\mathrm{R}^{2} 0,10\right)$. 
$\underline{V}$-Discussão

32 


\section{População do Instituto da Criança}

O Instituto da Criança é um hospital pediátrico de alta complexidade. As crianças atendidas na UTIP, em sua grande maioria, são pacientes com doenças de base, principalmente oncológicas e hepáticas, acompanhados nas especialidades pediátricas do próprio Instituto [31, 32]. Portanto, este trabalho não representa a prática ventilatória utilizada em crianças com patologias agudas, sem doença de base, como as estudadas em trabalhos prévios, mas potencialmente se aproxima do universo vivenciado nas UTIPs de hospitais de referência nível 3 do SUS [30].

\section{Prática da ventilação mecânica}

Neste trabalho, $35,7 \%$ dos pacientes admitidos na UTIP foram submetidos à VPM por 24 horas ou mais, o que condiz com a literatura [6]. Os pacientes que utilizaram VPM por menos de 24 horas foram aqueles em pósoperatório imediato, ou com rebaixamento de nível de consciência por condição orgânica (p. ex.: hemorragia), infecção de sistema nervoso central e distúrbio metabólico, além dos que foram a óbito antes de 24 horas da admissão.

A principal indicação de VPM foi a insuficiência respiratória aguda $(63,2 \%)$, geralmente associada a sepse grave / choque séptico $(61,3 \%$ dos pacientes com insuficiência respiratória aguda).

Em um grande trabalho multicêntrico, com dados coletados também na América Latina (mas não no Brasil), as modalidades ventilatórias mais utilizadas foram: ventilação mandatória intermitente sincronizada (SIMV) com 
ou sem pressão de suporte (39\%), pressão-controlada (25\%), ventilação assistido/ controlada a volume (23\%) e ventilação de alta freqüência por oscilação $(1,5 \%)$ [6]. Devemos ter em mente que em 1999, época em que tais dados foram coletados, algumas modalidades ventilatórias, hoje no mercado, ainda não estavam disponíveis, tais como a ventilação controlada a volume e regulada a pressão (PRVC) e volume de suporte.

As modalidades ventilatórias mais utilizadas em nosso estudo foram PS + SIMV e pressão controlada (PCV). O critério para o uso da PCV foi a maior gravidade do paciente, quando maior sua necessidade de "repouso" da musculatura respiratória $[3,7,33]$. Esta justificativa também se aplica ao uso de duas modalidades ventilatórias no mesmo paciente, conforme a evolução favorável da doença.

Como foi utilizada ventilação à pressão, atentou-se mais ao limite de pressão inspiratória (nunca maior que 30 ao início da VPM) do que propriamente ao controle do volume corrente, mesmo estando disponível a medida dos mesmos. Ao não se limitar o volume corrente, houve hiperventilação e hipocapnia.

Apesar da ventilação limitada à pressão ser de uso corrente em nosso meio, tanto pelo conceito de que altos picos inspiratórios levariam a barotrauma I pneumotórax, quanto pela simplicidade de seu uso, não podemos controlar o volume corrente a cada respiração, o qual pode sofrer grandes variações por maior ou menor escape da cânula traqueal, geralmente sem balão insuflável ("cuff") e por tudo que alterar a resistência ou a complacência do sistema.

Uma alternativa teoricamente vantajosa seria a ventilação controlada a volume e regulada a pressão (PRVC), onde determinamos o volume corrente a 
ser entregue sob a menor pressão possível, variando fluxo e pressão respiração a respiração. Este modo estaria bem indicado naqueles pacientes com mudanças abruptas na complacência e na resistência das vias aéreas, por exemplo, como ocorre no neonato após uso de surfactante ou no asmático após uso de broncodilatador [5, 33].

A pressão de suporte com SIMV é amplamente empregada em Pediatria, sendo eficaz em manter ou reativar a respiração espontânea sem gerar alterações hemodinâmicas, possíveis na ventilação controlada. Entretanto, por ser um modo pressórico, também falha em manter o volume corrente constante [5, 33].

Com conceito semelhante ao da PRVC, já temos no mercado aparelhos com volume de suporte (PRVS), bem indicado para desmame do aparelho.

A ventilação oscilatória de alta freqüência (HFOV) também apresenta vantagens teóricas como estratégia ventilatória: manutenção das vias aéreas abertas, menor volume corrente - entre 1 e $3 \mathrm{ml} / \mathrm{kg}$, trocas gasosas a pressões significativamente baixas, menor envolvimento do sistema cardiovascular, menor diminuição da produção endógena de surfactante.

\section{Pacientes com síndrome do desconforto respiratório agudo (SDRA)}

$\mathrm{Na}$ década de 70 até fins dos anos 90, eram utilizados volumes correntes suprafisiológicos (> 10ml/kg), pressões positivas ao final da expiração (PEEP) baixas, com uma alta mortalidade. Ao longo do tempo, todo o aparato tecnológico das unidades de terapia intensiva evoluiu, mas, mais do 
que respiradores melhores, foram introduzidas à prática clínica melhores estratégias ventilatórias, com menores volumes correntes e maiores PEEP.

Inicialmente em neonatos e depois em pacientes asmáticos, foi sendo desenvolvida a estratégia da hipercapnia permissiva, consolidada nos estudos sobre síndrome do desconforto respiratório agudo (SDRA) de Amato [23] e do ARDSNet [24] . É particularmente eficaz na SDRA por sepse, pois a acidose hipercápnica inibe a ativação do NFkB induzido por endotoxinas, diminuindo o processo inflamatório [34]. Esta estratégia protetora tem objetivos ventilatórios menos agressivos $\left(\mathrm{PaO}_{2}\right.$ entre 50 e 60 e $\mathrm{PaCO}_{2}$ em torno de 50 ou mais, desde que o pH esteja igual ou superior a 7,20) e visa basicamente "abrir os pulmões e mantê-los abertos".

Em ventilação volume-controlada, o volume corrente baseia-se na patologia pulmonar. Para pacientes com lesão pulmonar aguda, recomenda-se o emprego de um volume corrente em torno de $6 \mathrm{ml} / \mathrm{kg}$ de peso. Já a PEEP deve ser alta o suficiente para manter o recrutamento alveolar durante todo o ciclo respiratório e evitar lesão de estiramento; mas, por outro lado, não alta o bastante para causar alterações hemodinâmicas ou acidose hipercápnica acentuada $(\mathrm{pH}<7,20)[7,16,33]$.

Em situações extremas, geralmente em casos graves de SDRA, pode-se lançar mão de estratégias não habituais, ainda de baixo nível de evidência científica na população pediátrica, tais como o uso de óxido nítrico inalatório [35-37], administração de surfactante por via endotraqueal ou broncoscópica [38-43], manobras de recrutamento alveolar [44-47], posição prona [48, 49], insuflação traqueal de gases (TGI) em situações de hipercapnia [50], além da ventilação oscilatória de alta freqüência [29, 51]. 
Nos trabalhos dos grupos PALISI [22] e IGMVC [6], a incidência de SDRA relatada como diagnóstico no prontuário foi de $7,6 \%$ e $2 \%$, respectivamente. Em nosso estudo, 25 pacientes apresentaram critérios de SDRA, totalizando $10,37 \%$ do total de admissões na UTIP no mesmo período. Entretanto, nos trabalhos citados houve um grande número de pacientes não diagnosticados como SDRA, mas que apresentaram uma relação $\mathrm{PaO}_{2} / \mathrm{FiO}_{2}<$ 200 por um período prolongado de tempo, sugerindo um possível subdiagnóstico. Além disso, dado o perfil prospectivo de nosso trabalho, o diagnóstico de SDRA se deu por busca ativa, o que pode ter levado a uma maior acurácia.

Para nossos pacientes com LPA / SDRA, os parâmetros ventilatórios, tanto à admissão quanto em 48h de VPM, ainda não contemplam totalmente a estratégia de ventilação protetora, amplamente difundida na unidade. Quando foram instituídos PEEPs mais elevadas, maiores pressões médias de vias aéreas e volumes correntes mais baixos, por muitas vezes foram utilizadas freqüências respiratórias elevadas para a idade, o que não apenas impediu o desenvolvimento de hipercapnia permissiva, como fez com que 18 pacientes, ventilados há 48 horas, apresentassem hiperventilação.

Em relação às terapias de resgate para SDRA, não houve utilização de óxido nítrico inalatório ou de surfactante exógeno, já que estes não se encontravam disponíveis na unidade. A ventilação oscilatória de alta freqüência foi empregada precocemente (entre 24 e 48 horas após a intubação), mas apenas em dois pacientes durante o período do estudo. Além de só estar disponível um único ventilador de alta freqüência, seu manejo ainda estava sendo protocolado, sendo sua prática pouco usual. A insuflação traqueal de 
gases foi utilizada naqueles pacientes com hipercapnia não responsiva aos ajustes ventilatórios, com resposta insatisfatória. Já a posição prona foi utilizada aleatoriamente, sem mensuração do tempo em que cada paciente permaneceu em decúbito ventral, não sendo possível avaliar sua eficácia.

\section{Fatores de risco e desfechos}

* População geral (n=49)

A necessidade de ventilação mecânica prolongada se encontra associada a um maior desenvolvimento de complicações, infecciosas ou não, tais como pneumonia e pneumotórax [15, 52]. Portanto, o número de dias sob VPM tornase um importante desfecho de morbidade. Relação $\mathrm{PaO}_{2} / \mathrm{FiO}_{2}$ menor que 300 e volume corrente maior que $9 \mathrm{ml} / \mathrm{kg}$ de peso estão associados a períodos mais prolongados de ventilação mecânica [53].

Embora em nossa análise univariada, a relação $\mathrm{PaO}_{2} / \mathrm{FiO}_{2}$ inicial tenha sido fator de risco para VPM superior a 7 dias, apenas a pressão inspiratória inicial foi preditiva de tal duração de VPM na análise multivariada. Já na população com LPA, a relação $\mathrm{PaO}_{2} / \mathrm{FiO}_{2}$ em 48h de VPM foi o único fator preditivo para VPM prolongada.

Pacientes adultos sob ventilação mecânica, sem SDRA, têm como fatores de risco para o desenvolvimento de SDRA e, consequentemente, maior mortalidade, o emprego de grandes volumes correntes e de pressão inspiratória elevada [54]. Em nosso trabalho, também identificamos a pressão inspiratória como fator de risco para mortalidade, tanto na análise univariada, quanto na multivariada. 


\section{* Subpopulação com LPA}

Em pacientes com LPA /SDRA, já foram identificados como fatores de risco para mortalidade e/ou ventilação mecânica prolongada: $\mathrm{pH}$ inicial $[55,56]$, relação $\mathrm{PaO} 2 / \mathrm{FiO} 2[56,57]$, volumes correntes superiores a $8 \mathrm{ml} / \mathrm{kg}[23,24$, 56], PEEP inicial [56], pressão inspiratória e índice de oxigenação iniciais [56].

Em nossa população, identificamos como fatores de risco a pressão inspiratória inicial, como também $\mathrm{pH}$, relação $\mathrm{PaO} 2 / \mathrm{FiO} 2$ e índice de oxigenação em 48h de VPM. Não houve diferença quanto a PEEP e volume corrente entre sobreviventes e não-sobreviventes.

\section{Subutilização da ventilação protetora}

Diversos autores vêm tentando explicar os motivos da subutilização da VPM protetora, em estudos realizados com pacientes adultos: desconforto do paciente, taquipnéia, hipercapnia, acidose, piora da oxigenação, não reconhecimento da doença e equipe de assistência desconfortável com o emprego de baixos volumes correntes (principalmente pelo risco de atelectasia) [58-62].

No caso da UTIP do Instituto da Criança, todo o corpo clínico é bem familiarizado tanto com o diagnóstico, quanto com a terapêutica adequada para LPA/ SDRA. Embora haja respiradores adequados para implementação e controle de uma estratégia ventilatória protetora, não há um protocolo escrito de tratamento, o que permite variações de estratégias ventilatórias, conforme o 
médico assistente. Também é possível que $\mathrm{PaCO}_{2}$ elevadas gerem desconforto na equipe de assistência, o que faz com que as freqüências respiratórias sejam elevadas, de forma compensatória.

Wolthuis e colaboradores estudaram o volume corrente utilizado em três UTIs de pacientes adultos na Holanda, com o intuito de determinar o impacto de educação e orientações após a apresentação de dados da prática clínica ("feedback and education"), em relação ao uso da ventilação protetora. Inicialmente, o volume corrente utilizado era de $9,8 \mathrm{ml} / \mathrm{kg} \pm 2,0$. Seis meses após a apresentação dos dados à equipe de assistência, e educação sobre a importância da ventilação protetora, os dados foram novamente coletados e o volume corrente médio era de $8,1 \mathrm{ml} / \mathrm{kg} \pm 1,7$. Este estudo mostra a importância da realização de trabalhos que demonstrem a prática clínica corrente, visando seu aprimoramento através de iniciativas de educação continuada [63].

Sugerimos o uso de um protocolo escrito para VPM em pacientes com LPA/SDRA, assim como a apresentação periódica da prática em vigor através de programas de educação continuada. Assim, talvez possamos maximizar o uso da ventilação protetora nestes pacientes. 
VI-Conclusões 
- A observação da prática da ventilação mecânica nas crianças admitidas na UTIP do Instituto da Criança revelou uma prevalência de ventilação a pressão.

- Dos fatores de risco investigados no total da população estudada, a pressão inspiratória inicial, $\mathrm{pH}$, relação $\mathrm{PaO} 2 / \mathrm{FiO} 2$ e índice de oxigenação iniciais, assim como o índice de oxigenação em 48h de VPM foram significativos para mortalidade. Somente a pressão inspiratória inicial foi preditiva de VPM superior a sete dias.

- Na subpopulação de pacientes com lesão pulmonar aguda, pressão inspiratória inicial, pH, índice de oxigenação e relação PaO2/FiO2 em 48h de VPM foram fatores preditivos para mortalidade, mas somente este último foi preditivo de VPM prolongada.

- A estratégia de ventilação protetora para pacientes com LPA / SDRA está sendo subutilizada. A implementação de um protocolo para VPM em LPA/SDRA talvez possa maximizar o uso de tal estratégia, assim como a apresentação periódica da prática em vigor através de programas de educação continuada. 


\section{VII-Referências Bibliográficas}


1. World Health Organization Statistical Information System. Disponível em http://apps.who.int/whosis/data. Acessado em 20/08/2009.

2. Benicio, M.H., et al., [Secular trends in respiratory diseases of childhood in the city of Sao Paulo, Brazil (1984-1996)]. Rev Saude Publica, 2000. 34(6 Suppl): p. 91-101.

3. Carvalho, W.B., Oliveira, N.F., Santana, J.C.B., Piva, J.P., Garcia, P.C.R., Insuficiência Respiratória, in Medicina Intensiva em Pediatria, J.P. Piva, Garcia, P.C.R., Editor. 2005, Revinter: Rio de Janeiro. p. 363375.

4. Grumach, A.S., Kanarek, D.A.M., Características da Resposta Imune na Criança, in Pediatria Básica, E. Marcondes, Vaz, F.A.C., Ramos, J. L. A., Okay, Y., Editor. 2003, Sarvier: São Paulo. p. 833-836.

5. Matsumoto, T., Ventilação Mecânica, in Atualizações em Terapia Intensiva Pediátrica, J.H. Jyh, Nóbrega, R.F., Souza, R.L., Editor. 2007, Atheneu: São Paulo. p. 201-218.

6. Farias, J.A., et al., What is the daily practice of mechanical ventilation in pediatric intensive care units? A multicenter study. Intensive Care Med, 2004. 30(5): p. 918-25.

7. Cheifetz, I.M., Invasive and noninvasive pediatric mechanical ventilation. Respir Care, 2003. 48(4): p. 442-53; discussion 453-8.

8. Goldstein, B., Giroir, B., Randolph,A. International pediatric sepsis consensus conference: definitions for sepsis and organ dysfunction in pediatrics. Pediatr Crit Care Med, 2005. 6(1): p. 2-8. 
9. de Azevedo, Z.M., et al., [Children with acute respiratory distress syndrome: anatomoclinical and radiologic correlation]. Rev Soc Bras Med Trop, 1999. 32(5): p. 557-70.

10. Pinheiro, B.V., et al. Accuracy of clinical diagnosis of acute respiratory distress syndrome in comparison with autopsy findings. J Bras Pneumol, 2007. 33(4): p. 423-8.

11. Ware, L.B. Pathophysiology of acute lung injury and the acute respiratory distress syndrome. Semin Respir Crit Care Med, 2006. 27(4): p. 337-49.

12. Bernard, G.R., et al., The American-European Consensus Conference on ARDS. Definitions, mechanisms, relevant outcomes, and clinical trial coordination. Am J Respir Crit Care Med, 1994. 149(3 Pt 1): p. 818-24.

13. Carpi, M.F., Fioretto, J.R., Síndrome do Desconforto Respiratório Agudo, in Atualizações em Terapia Intensiva Pediátrica, J.H. Jyh, Nóbrega, R.F., Souza, R.L., Editor. 2007, Atheneu: São Paulo. p. 141-156.

14. Troster, E.J., Faria, L.S., Síndrome de desconforto respiratório agudo em crianças, in Terapia Intensiva: Pediatria e Neonatologia, A. Stape, Troster, E.J., Deutsch, A.D., Editor. 2005, Atheneu: São Paulo. p. 83100.

15. Silva Filho, L.V.F., Bozelli, F.C., Piacentini, A.P.S., Carvalho, W.B., Complicações da Ventilação Pulmonar Mecânica, in Ventilação Pulmonar Mecânica em Pediatria e Neonatologia, W.B. Carvalho, Hirschheimer, M.R., Proença Filho, J.O., Freddi, N.A., Troster, E.J., Editor. 2005, Atheneu: São Paulo. p. 543-552. 
16. Chiumello, D., et al., Lung stress and strain during mechanical ventilation for acute respiratory distress syndrome. Am J Respir Crit Care Med, 2008. 178(4): p. 346-55.

17. Gattinoni, L., P. Caironi, and E. Carlesso, How to ventilate patients with acute lung injury and acute respiratory distress syndrome. Curr Opin Crit Care, 2005. 11(1): p. 69-76.

18. Esteban, A., et al., How is mechanical ventilation employed in the intensive care unit? An international utilization review. Am J Respir Crit Care Med, 2000. 161(5): p. 1450-8.

19. Amato, M.B., et al., [Mechanical ventilation in Acute Lung Injury (ALI)/Acute Respiratory Discomfort Syndrome (ARDS)]. J Bras Pneumol, 2007. 33 Suppl 2S: p. S119-27.

20. Sarnaik, A.P., et al., Pressure-controlled ventilation in children with severe status asthmaticus. Pediatr Crit Care Med, 2004. 5(2): p. 133-8.

21. Sachdev, A., Chugh, K., Gupta, D., Agarwal, S., Comparision of two ventilation modes and their clinical implications in sick children. Indian Journal of Critical Care Medicine, 2005. 9(4): p. 205-210.

22. Randolph, A.G., et al., The feasibility of conducting clinical trials in infants and children with acute respiratory failure. Am J Respir Crit Care Med, 2003. 167(10): p. 1334-40.

23. Amato, M.B., et al., Effect of a protective-ventilation strategy on mortality in the acute respiratory distress syndrome. N Engl J Med, 1998. 338(6): p. 347-54.

24. The Acute Respiratory Distress Syndrome Network. Ventilation with lower tidal volumes as compared with traditional tidal volumes for acute 
lung injury and the acute respiratory distress syndrome. N Engl J Med, 2000. 342(18): p. 1301-8.

25. Balcells Ramirez, J., J. Lopez-Herce Cid, and V. Modesto Alapont, [Prevalence of mechanical ventilation in pediatric intensive care units in Spain]. An Pediatr (Barc), 2004. 61(6): p. 533-41.

26. Fedora, M., et al., [Mechanical ventilation on paediatric intensive care units in Czech Republic]. Anasthesiol Intensivmed Notfallmed Schmerzther, 2005. 40(3): p. 173-8.

27. Kendirli, T., et al., Mechanical ventilation in children. Turk J Pediatr, 2006. 48(4): p. 323-7.

28. Rossi, F.S., et al., Utilization of the lower inflection point of the pressurevolume curve results in protective conventional ventilation comparable to high frequency oscillatory ventilation in an animal model of acute respiratory distress syndrome. Clinics (Sao Paulo), 2008. 63(2): p. 23744.

29. Playfor, S.D., The role of high-frequency oscillatory ventilation in paediatric intensive care. Crit Care, 2005. 9(3): p. 249-50.

30. Saúde, M.d.E.d., Portaria $n^{\circ}$ 2.224/GM - Classificação Hospitalar do Sistema Único de Saúde. 2002: Brasília.

31. de Oliveira, C.F., et al., ACCM/PALS haemodynamic support guidelines for paediatric septic shock: an outcomes comparison with and without monitoring central venous oxygen saturation. Intensive Care Med, 2008. 34(6): p. 1065-75. 
32. Cardoso, M.P., et al., Comparison between clinical diagnoses and autopsy findings in a pediatric intensive care unit in Sao Paulo, Brazil. Pediatr Crit Care Med, 2006. 7(5): p. 423-7.

33. Freddi, N.A., Silva, D.C.B., Ventilação Protetora no Paciente Pediátrico, in Atualizações em Terapia Intensiva Pediátrica, J.H. Jyh, Nóbrega, R.F., Souza, R.L., Editor. 2007, Atheneu: São Paulo. p. 219-228.

34. Takeshita, K., et al., Hypercapnic acidosis attenuates endotoxin-induced nuclear factor-[kappa]B activation. Am J Respir Cell Mol Biol, 2003. 29(1): p. 124-32.

35. Fioretto, J.R., et al., Acute and sustained effects of early administration of inhaled nitric oxide to children with acute respiratory distress syndrome. Pediatr Crit Care Med, 2004. 5(5): p. 469-74.

36. Michael, J.R., et al., Inhaled nitric oxide versus conventional therapy: effect on oxygenation in ARDS. Am J Respir Crit Care Med, 1998. 157(5 Pt 1): p. 1372-80.

37. Dobyns, E.L., et al., Multicenter randomized controlled trial of the effects of inhaled nitric oxide therapy on gas exchange in children with acute hypoxemic respiratory failure. J Pediatr, 1999. 134(4): p. 406-12.

38. Gregory, T.J., et al., Bovine surfactant therapy for patients with acute respiratory distress syndrome. Am J Respir Crit Care Med, 1997. 155(4): p. $1309-15$.

39. Gunther, A., et al., Surfactant alteration and replacement in acute respiratory distress syndrome. Respir Res, 2001. 2(6): p. 353-64.

40. Anzueto, A., et al., Aerosolized surfactant in adults with sepsis-induced acute respiratory distress syndrome. Exosurf Acute Respiratory Distress 
Syndrome Sepsis Study Group. N Engl J Med, 1996. 334(22): p. 141721.

41. Walmrath, D., et al., Bronchoscopic administration of bovine natural surfactant in ARDS and septic shock: impact on gas exchange and haemodynamics. Eur Respir J, 2002. 19(5): p. 805-10.

42. Willson, D.F., et al., Effect of exogenous surfactant (calfactant) in pediatric acute lung injury: a randomized controlled trial. JAMA, 2005. 293(4): p. 470-6.

43. Willson, D.F., P.R. Chess, and R.H. Notter, Surfactant for pediatric acute lung injury. Pediatr Clin North Am, 2008. 55(3): p. 545-75, ix.

44. Barbas, C.S., et al., Mechanical ventilation in acute respiratory failure: recruitment and high positive end-expiratory pressure are necessary. Curr Opin Crit Care, 2005. 11(1): p. 18-28.

45. Brower, R.G., et al., Effects of recruitment maneuvers in patients with acute lung injury and acute respiratory distress syndrome ventilated with high positive end-expiratory pressure. Crit Care Med, 2003. 31(11): p. 2592-7.

46. Moran, I., et al., Recruitment manoeuvres in acute lung injury/acute respiratory distress syndrome. Eur Respir J Suppl, 2003. 42: p. 37s-42s.

47. Gaudêncio, A.M.A.S., Barbas, C.S.V., Troster, E.J., Recrutamento Pulmonar, in Ventilação Pulmonar Mecânica em Pediatria e Neonatologia, W.B. Carvalho, Hirschheimer, M.R., Proença Filho, J.O., Freddi, N.A., Troster, E.J., Editor. 2005, Atheneu: São Paulo. p. 33-40.

48. Gattinoni, L., Protti, A. Ventilation in the prone position: for some but not for all? CMAJ, 2008. 178(9): p. 1174-6. 
49. Marraro, G.A. Innovative practices of ventilatory support with pediatric patients. Pediatr Crit Care Med, 2003. 4(1): p. 8-20.

50. Hoffman, L.A. Novel strategies for delivering oxygen: reservoir cannula, demand flow, and transtracheal oxygen administration. Respir Care, 1994. 39(4): p. 363-77; discussion 386-9.

51. Rimensberger, P.C., et al. First intention high-frequency oscillation with early lung volume optimization improves pulmonary outcome in very low birth weight infants with respiratory distress syndrome. Pediatrics, 2000. 105(6): p. $1202-8$.

52. Rivera, R., Tibballs, J. Complications of endotracheal intubation and mechanical ventilation in infants and children. Crit Care Med, 1992. 20(2): p. 193-9.

53. Halbertsma, F.J., et al. The oxygenation ratio during mechanical ventilation in children: the role of tidal volume and positive end-expiratory pressure. J Crit Care, 2009. 24(2): p. 220-6.

54. Jia, X., et al. Risk factors for ARDS in patients receiving mechanical ventilation for $>48 \mathrm{~h}$. Chest, 2008. 133(4): p. 853-61.

55. Flori, H.R., et al. Pediatric acute lung injury: prospective evaluation of risk factors associated with mortality. Am J Respir Crit Care Med, 2005. 171(9): p. 995-1001.

56. Erickson, S., et al. Acute lung injury in pediatric intensive care in Australia and New Zealand: a prospective, multicenter, observational study. Pediatr Crit Care Med, 2007. 8(4): p. 317-23.

57. Dahlem, P., et al. Incidence and short-term outcome of acute lung injury in mechanically ventilated children. Eur Respir J, 2003. 22(6): p. 980-5. 
58. Mikkelsen, M.E., et al. Potential reasons why physicians underuse lungprotective ventilation: a retrospective cohort study using physician documentation. Respir Care, 2008. 53(4): p. 455-61.

59. Kalhan, R., et al. Underuse of lung protective ventilation: analysis of potential factors to explain physician behavior. Crit Care Med, 2006. 34(2): p. 300-6.

60. Rubenfeld, G.D., et al. Barriers to providing lung-protective ventilation to patients with acute lung injury. Crit Care Med, 2004. 32(6): p. 1289-93.

61. Wongsurakiat, P., D.J. Pierson, and G.D. Rubenfeld. Changing pattern of ventilator settings in patients without acute lung injury: changes over 11 years in a single institution. Chest, 2004. 126(4): p. 1281-91.

62. Young, M.P., et al. Ventilation of patients with acute lung injury and acute respiratory distress syndrome: has new evidence changed clinical practice? Crit Care Med, 2004. 32(6): p. 1260-5.

63. Wolthuis, E.K., et al. Feedback and education improve physician compliance in use of lung-protective mechanical ventilation. Intensive Care Med, 2005. 31(4): p. 540-6. 
VIII-Anexos 
Anexo 1 - Formulário de coleta dos dados

\section{VENTILAÇÃO MECÂNICA EM PEDIATRIA}

NOME:

DATA DE NASCIMENTO:

PESO: $K G$

PRONTUÁRIO:

DOENÇA DE BASE:

DIAGNÓSTICO ATUAL:

PELOD:

TERAPÊUTICA INSTITUÍDA:

$\beta 2$ inalatório

$\beta 2 \mathrm{EV}$

corticoterapia inalatória

corticoterapia EV. Qual / dose

oxigenoterapia inalatória. ( )cateter ( )máscara ( )oxyhood/tenda

ACESSO À VIA AÉREA:

intubação orotraqueal

intubação nasotraqueal

máscara: ( )nasal ( )orofacial

traqueostomia (data cirurgia:

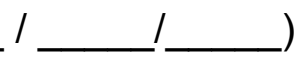

RESPIRADOR UTILIZADO:

INDICAÇÃO DA VPM (somente 1 dos grupos, a causa predominante):

exacerbação aguda de doença pulmonar crônica: displasia

broncopulmonar, fibrose cística, malformações torácicas ou pulmonares, necessitando vpm por infecção, broncoespasmo ou outro episódio agudo.

rebaixamento do nível de consciência: por condição orgânica (p.ex.:

hemorragia snc), infecção (p.ex.: meningite), distúrbio hidroeletrolítico (p.ex.: hipernatremia)

doença neuromuscular: doenças e desordens nervos periféricos, junção mioneural e músculos (inclui guillain-barrè)

falência respiratória aguda

SE FALÊNCIA RESPIRATÓRIA AGUDA, POR (aceita mais de 1 resposta):

PNEUMONIA SEM SEPSE

BRONQUIOLITE

BRONCOASPIRAĈ̃O

PÓS OPERATÓRIO

INSUFICIÊNCIA CARDÍACA

SEPSE / CHOQUE SÉPTICO PULMONAR

SEPSE / CHOQUE SÉPTICO NÃO PULMONAR 
OBSTRUÇÃO VAS

SDRA

OUTROS:

DADOS VENTILATÓRIOS:

\begin{tabular}{|l|l|l|l|l|l|}
\hline DATA & D1 VPM & D2 & D3 & D4 & D5 \\
\hline MODO & & & & & \\
VENT & & & & & \\
\hline PINSP & & & & & \\
\hline VC & & & & \\
(ML/KG) & & & & & \\
\hline PEEP & & & & & \\
\hline MAP & & & & & \\
\hline T insp & & & & & \\
\hline FR & & & & & \\
\hline FIO2 & & & & & \\
\hline PH & & & & & \\
\hline PACO2 & & & & & \\
\hline PAO2 & & & & & \\
\hline BIC & & & & & \\
\hline BE & & & & & \\
\hline SatO2 & & & & & \\
\hline CONDUTA & & & & & \\
\hline
\end{tabular}

OBSERVAÇÕES:

FALÊNCIA DE ÓRGÃOS E SISTEMAS:

ACV

Respiratório

Hemato

Hepato

Nefro

SNC

Outro:

DESFECHOS:

Dias de VPM:

Dias de internação na UTI:

Dias de internação hospitalar:

Mortalidade na UTI:

Mortalidade hospitalar: 


\section{Anexo 2 - Aprovação do projeto junto à Comissão de Ética}

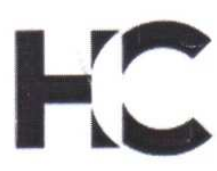

\section{APROVAÇÃO}

A Comissão de Ética para Análise de Projetos de Pesquisa - CAPPesq da Diretoria Clínica do Hospital das Clínicas e da Faculdade de Medicina da Universidade de São Paulo, em sessão de 28.07.05, APROVOU o Protocolo de Pesquisa $n^{\circ} 333 / 05$, intitulado: "Prática de Ventilação Mecânica em Unidade de Terapia Intensiva Pediátrica de Hospital Terciário" apresentado pelo Departamento de PEDIATRIA, inclusive Termo de Consentimento Livre e Esclarecido.

Cabe ao pesquisador elaborar e apresentar à CAPPesq, os relatórios parcial e final sobre a pesquisa (Resolução do Conselho Nacional de Saúde $n^{\circ} 196$, de 10.10.1996, inciso IX.2, letra "c")

Pesquisador(a) Responsável: Dr Eduardo Juan Troster

Pesquisador(a) Executante: Dra Dáfne Cardoso Bourguignon da Silva

CAPPesq, 28 de Julho de 2005.

PROF. DR. CLAUDIO LEONE

Vice-Presidente da Comissão de Ética para Análise de Projetos de Pesquisa 


\section{Anexo 3 - Certificado de apresentação de tema livre no VIII Congresso}

\section{Panamericano e Ibérico de Medicina Crítica e Terapia Intensiva}

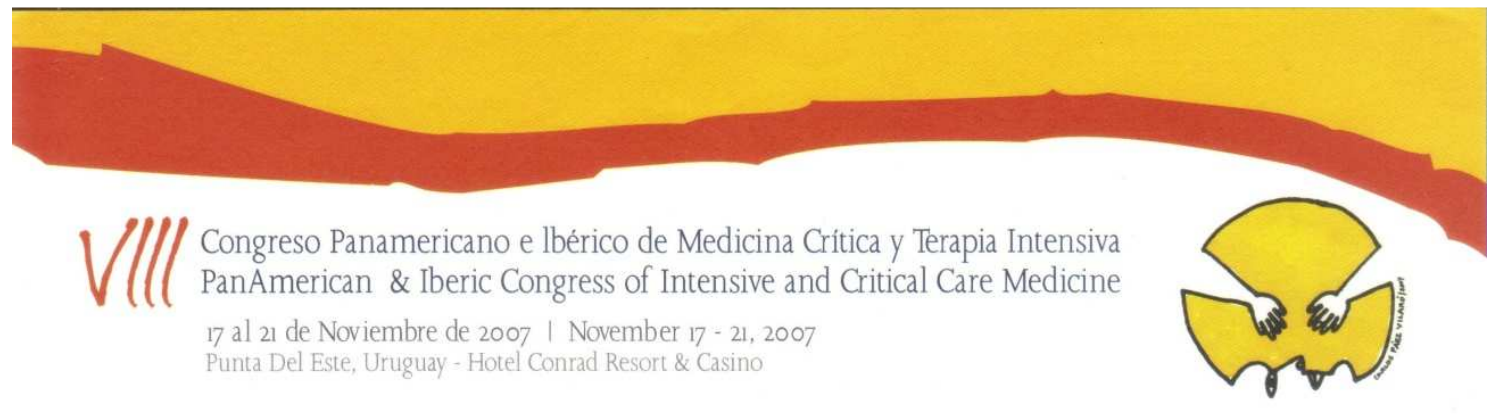

Punta del Este, November 2007

We hereby certify that the scientific paper:

\section{“MECHANICAL VENTILATION PROFILE OF A BRAZILIAN PEDIATRIC INTENSIVE CARE UNIT HOW WE ARE VENTILATING OUR CHILDREN"}

Authors:

"Bourguignon da Silva, DC; Shibata, A R O; Ferraro, A A; Troster, E J."

was presented at the VIII Pan-American \& Iberic Congress of Intensive and Critical Care Medicine, held at the Conrad Resort \& Casino, Punta del Este, Uruguay, from 17 to 21 November, 2007.

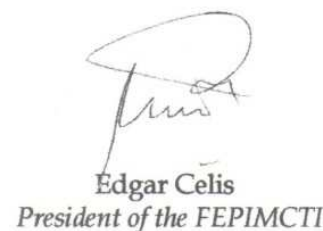

President of the FEPIMCTI
Hernán Artucio
President of the Organizing Committee

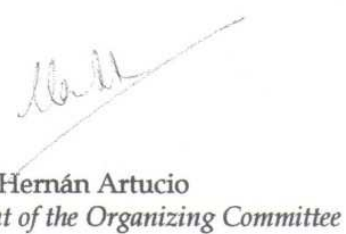

Montevideo - Uruguay

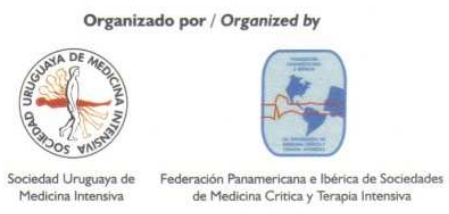


Anexo 4 - Artigo "How is mechanical ventilation employed in a pediatric intensive care unit in Brazil?", aceito para publicação na revista "Clinics"

\title{
HOW IS MECHANICAL VENTILATION EMPLOYED IN A PEDIATRIC INTENSIVE CARE UNIT IN BRAZIL?
}

Running head: Mechanical Ventilation in a PICU in Brazil

\begin{abstract}
OBJECTIVE: to investigate the relationship between mechanical ventilation and mortality and the practice of mechanical ventilation applied in children admitted to a high-complexity pediatric intensive care unit (PICU) in the city of São Paulo, Brazil
\end{abstract}

DESIGN: Prospective cohort study of all consecutive patients admitted to a Brazilian high-complexity PICU who were placed on MV for 24 hours or more, between October $1^{\text {st }}, 2005$ and March 31 ${ }^{\text {st }}, 2006$.

RESULTS: Of the 241 patients admitted, 86 (35.7\%) received MV for 24 hours or more. Of these, 49 met inclusion criteria and were thus eligible to participate in the study. Of the 49 patients studied, 45 had chronic functional status. The median age of participants was 32 months and the median length of MV use was 6.5 days. The major indication for MV was acute respiratory failure, usually associated with severe sepsis / septic shock. Pressure ventilation modes were the standard ones. An overall 10.37\% incidence of Acute Respiratory Distress Syndrome (ARDS) was found, in addition to tidal volumes $>8 \mathrm{ml} / \mathrm{kg}$, as well as normo- or hypocapnia. A total of 17 children died. Risk factors for mortality 
within 28 days of admission were initial inspiratory pressure, $\mathrm{pH}, \mathrm{PaO} 2 / \mathrm{FiO} 2$ ratio, oxygenation index and also oxygenation index at 48 hours of MV. Initial inspiratory pressure was also a predictor of MV for periods longer than 7 days.

CONCLUSION: Of the admitted children, $35.7 \%$ received MV for $24 \mathrm{~h}$ or more. Pressure ventilation modes were standard. Of the children studied, $91 \%$ had chronic functional status. There was a high incidence of ARDS, but a lungprotective strategy was not fully implemented. Inspiratory pressure at the beginning of $\mathrm{MV}$ was a predictor of mortality within 28 days and a longer course of MV.

KEYWORDS: Mechanical ventilation, intensive care units, risk factors, children, health profile. 


\section{INTRODUCTION}

Diseases of the respiratory system are highly prevalent among Brazilian children, especially those aged less than 5 years. The World Health Organization Statistical Information System (WHOSIS) reported that Brazilian under-5 mortality rate, in 2000 , was still $30 / 1000$ live births. $13 \%$ of them have died from pneumonia alone. ${ }^{1}$

Specifically in the city of São Paulo, there is an increase of respiratory diseases. While in the 80 's $29 \%$ of children younger than age 5 presented respiratory disease, with or without wheezing, in the 90's the number increased to $49.6 \%{ }^{2}$

In many cases of respiratory diseases the use of ventilatory support is required. Besides that, ventilatory support may be needed in other situations, such as in sepsis and septic shock, neuromuscular diseases, during postoperative state and in cases of altered mental status with loss of consciousness. Thus, mechanical ventilation (MV) has become one of the major indications for admission to intensive care units.

Although MV benefits are unquestionable, its use can also cause harm. The adverse consequences of MV include airway lesions by intubation, pneumonia/sinusitis associated with MV, volutrauma, barotrauma, atelectrauma, and biotrauma. From the 70's until the late 90's, supraphysiologic tidal volumes $(>10 \mathrm{ml} / \mathrm{kg}$ ), and low or even zero positive end expiration pressures (PEEP) were used, with high mortality rates observed ${ }^{3}$. In the last years, technical devices in the intensive care units had improved and better ventilatory strategies have been introduced. 
According to the lung-protective ventilation strategy, tidal volumes lower than $8 \mathrm{ml} / \mathrm{kg}$, PEEP high enough to permit FiO2 less than 0.6 and oxygen saturation (SatO2) above $88 \%$, besides limiting plateau pressure, are indicated to achieve better outcomes, especially lower mortality rates, in patients with ARDS and ALI. ${ }^{4,5}$ Retrospective studies in infants and children suggest that the implementation of lung protective strategies could also be lowering the mortality rate. Albuali and coworkers showed that mortality decreased by $40 \%$ over the last 15 years, as lung-protective strategies have been used. In the study, higher tidal volume was independently associated with increased mortality and decreased ventilation-free days. ${ }^{6}$

Although data exist that 17 to $64 \%$ of all children admitted to PICUs have been mechanically ventilated, no epidemiologic study of this phenomena have been made in Brazilian children. ${ }^{7-11}$ Two multicenter studies, the International Group for Mechanical Ventilation in Children (IGMVC) and the Pediatric Acute Lung Injury and Sepsis Investigators (PALISI) showed that children on MV have a median age of 1 year, are ventilated for 6 or 7 days and frequently use more than one ventilatory mode. Prevalence of ARDS was low in both multicenter studies: $2 \%$ in the IGMVC study and $7.6 \%$ in the PALISI investigation. The PALISI trial also showed that $52.5 \%$ of children had a $\mathrm{PaO} 2 / \mathrm{FiO} 2$ ratio of 200 or less for at least one day and that some of these may represent underrecognized cases of ARDS. ${ }^{7,8}$

It is unclear whether these results apply to our population in Brazil, since our population may be different and the current practice of mechanical ventilation is not known. Thus, in this study we investigated the practice of mechanical ventilation adopted in children admitted to a high-complexity 
pediatric intensive care unit (PICU) in the city of São Paulo, Brazil. To investigate the relationship between mechanical ventilation and mortality, we searched for risk factors that may have affected mortality within 28 days of admission to the PICU, and days on MV.

\section{METHODS}

The "Instituto da Criança" is a reference hospital for high-complexity pediatric diseases and is affiliated with "Universidade de São Paulo". It is a 135bed pediatric hospital, with 13 beds in the PICU, at the time of our study.

The institutional review board approved the study before data collection began and waived informed consent, as the study was observational only. In order to collect data that were representative of current practice, the PICU medical team knew researchers had authorization to medical records, but only the PICU coordinator and the researchers were not blinded to what data were been collected.

All children admitted to the "Instituto da Criança" PICU from October $1^{\text {st }}$, 2005 to March $31^{\text {st }}$, 2006, who underwent MV for 24 hours or more, were included in the study. Exclusion criteria were the following: lack of arterial blood gas measurement, patients using ventilator devices without a tidal volume measurement, noninvasive ventilation, previous dependence upon $\mathrm{MV}$, and oxygenation impairment by heart disease or pulmonary hypertension. Patients ventilated in another institution for more than 24 hours and transferred to our PICU were also excluded from the study. Two patients were on high frequency 
oscillatory ventilation, after a time of pressure ventilation. Only data from pressure ventilation were analyzed in these two, because of the inability to compare ventilatory variables.

Demographic data (gender, age, weight), date of admission, chronic functional status, date of initiating MV, access to airway, reason for $M V$ and ventilator data were prospectively collected by two pediatric intensivists (DCBS, AROS), in forms based on that created by the IGMVC and adapted to our study. ${ }^{7}$ Exhaled tidal volume measured by the ventilator device was used, as not all endotracheal tubes were cuffed.

Table 1 lists all variables used in this study, as formerly used by the IGMVC Study. The variables were studied at two different times: T0 corresponded to the beginning of MV (first arterial blood gas measurement after MV start) and T1 to 48 hours post MV (arterial blood gas measurement closer to 48 hours of the institution of MV).

The Pediatric Logistic Organ Dysfunction (PELOD) was used as a prognostic index. ${ }^{12}$ The outcomes analyzed included days of MV and mortality within 28 days after admission. To complete the outcomes analysis, all medical records were reviewed after patients were discharged from the hospital.

Arterial blood punctures were done without topical anesthesia. However, rapid sequence intubation was routinely performed with pre-oxygenation, administration of a sedative agent, an analgesic, and a paralyzing agent. Thus, when the arterial blood puncture was made, patients had already received these agents for 30 minutes to two hours prior the procedure. It was also part of standard care to keep ventilated patients under continuous sedation and 
analgesia, usually with midazolam and fentanyl. Blood punctures were performed on patients after they had received these agents.

The standard ventilator devices of the PICU were used, in which, besides expiratory tidal volume measurement, the following modes were available: pressure control ventilation (PCV), volume control (VCV), volume target pressure control (VTPC), synchronized intermittent mandatory ventilation (SIMV) with pressure support (PS) and spontaneous (Newport E500®, Newport Medical Instruments, Inc., Newport Beach, CA, USA).

Statistical analysis was performed with StatView for Windows 5.01 software (SAS Institute, Cary, NC, USA). Categorical data are expressed as absolute counts and percentages. Continuous data are expressed as medians and interquartile range. Data were considered significant at $p<0.05$. The MannWhitney $U$ test was used for non-paired continuous data, for two groups (survivors $\mathrm{x}$ nonsurvivors). Risk factors were identified by simple regression (univariate analysis) and their association power was measured by their odds ratios, considering the respective $95 \%$ confidence intervals. 


\section{RESULTS}

A total of 241 patients were admitted to the PICU between October $1^{\text {st }}$, 2005 and March $31^{\text {st }}, 2006$, with 86 undergoing mechanical ventilation for 24 hours or more. Of the 86,37 met exclusion criteria, resulting in data from 49 eligible patients being analyzed (table 1). Participants had a median age of 32 months (P25; P75: 1.87; 62.12) and median weight of $13 \mathrm{~kg}$ (P25; P75: 7.77; 18.22). A large majority of the sample was male (61.23\%). No patient data was lost.

Only 4 of 49 patients $(8.16 \%)$ had no chronic status. The most prevalent chronic diseases were as follows: respiratory $(n=13 ; 26.53 \%)$, neurological $(n=9 ; 18.37 \%)$, hepatic $(n=6 ; 12.24 \%)$ and oncological $(n=7 ; 14.28 \%)$.

The major indication for MV in the 49 patients eligible for this study was acute respiratory failure $(n=29,59.18 \%)$, followed by acute decompensation of chronic pulmonary disease $(n=11,22.45 \%)$ and a lowered level of consciousness $(n=8,16.33 \%)$. Among patients with acute respiratory failure, $62 \%(n=17)$ had severe sepsis / septic shock.

The airway was achieved by orotracheal intubation, in most cases. There was no nasotracheal intubation. A total of 5 patients were previously tracheostomized, while another 3 were tracheostomized after 3 weeks of MV.

No child was ventilated by VCV. Of the total, 48 were ventilated by pressure modes and one was ventilated by volume-targeted pressure. A total of 23 patients $(47 \%)$ received only one ventilatory mode (PCV or PS with SIMV) and $26(53 \%)$ received more than one mode. 
Weaning and extubation were not focused in this study, as they are included in another investigation currently underway.

Although no patients had an inspiratory pressure above $30 \mathrm{cmH} 2 \mathrm{O}$ at T0, at T1 9 patients had it. At T1, 23 patients had a tidal volume of $10 \mathrm{ml} / \mathrm{kg}$ or more; instead of that, no barotrauma was observed (figure 1).

Most patients were normo or hypocapnic (figure 2). A total of 41 patients had clinical, $\mathrm{PaO} 2 / \mathrm{FiO} 2$ ratio and radiological data compatible with an ARDS diagnosis $(n=25)$ or with acute lung injury non-ARDS $(n=16)$, in the first 48 hours of MV (13).

A median PELOD score of 11 was calculated, which corresponded to a $1.3 \%$ theoretical mortality. However, 28 days after admission to the PICU, 17 patients had died (34.69\%).

As risk factors for mortality, we identified inspiratory pressure $(p=0.005)$, $\mathrm{pH}(p=0.049), \mathrm{PaO}_{2} / \mathrm{FiO}_{2}(p=0.016)$ and oxygenation index at T0 $(p=0,0007)$, as well as oxygenation index at T1 $(p=0.011)$. PEEP and TV at T0 were not detected as risk factors. Table 2 shows the odd ratio of each risk factor for mortality and for staying more than 7 days on MV.

Univariate analysis (simple regression) showed a correlation between days of MV and inspiratory pressure at T0 ( $\left.p 0.015 ; R^{2} 0.130\right)$, as also between

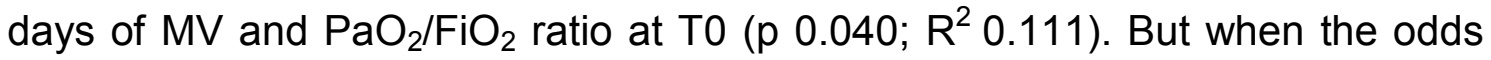
ratios were calculated, only initial inspiratory pressure was predictive of MV of more than 7 days, with an OR of 5.96 (IC 95\% 1.69 - 21.03) (table 2). 


\section{DISCUSSION}

The "Instituto da Criança" is a high-complexity pediatric hospital, affiliated with " Universidade de São Paulo". Children who are admitted to the PICU are usually chronically ill and treated by pediatric subspecialties from the institute itself. $^{14,15}$ Thus, one weakness of this study is it is not representative of the ventilatory practices of all Brazilian PICUs, but it may reflect the patterns of PICUs at other reference institutions in the country. Other weakness is that data extraction was performed around three years ago and practice could change in this time. Nevertheless, its major strength is to be the first Brazilian report of practice of mechanical ventilation in pediatric intensive care setting.

In this study, $35.7 \%$ of admitted patients underwent mechanical ventilation for 24 hours or more. This finding agrees with that described by others $^{7-11}$. Patients ventilated for less than 24 hours were usually of immediate postoperative status, those who had low level of consciousness that was rapidly reversed, and those who died within 24 hours after PICU admission.

The major indication for MV was acute respiratory failure (59.18\%), generally associated with severe sepsis/septic shock $(17 / 29,58.62 \%$ of children with acute respiratory failure).

As pressure based ventilatory modes were mostly used, even though other modes were available, we can assume that our standard ventilatory pattern is pressure ventilation. The most used modes were PS + SIMV and PCV. Criteria for using PCV were severity of illness-the more severe the illness, the greater the necessity for resting the respiratory muscles. 
As pressure ventilation was used, and no volume ventilation at all, we can assume more attention was paid to inspiratory pressure limits than to tidal volume control, even though both measures were available.

Concerning selection of ventilatory settings, it's difficult to compare them among patients with different pathologies ${ }^{7}$. So, we compare settings in ALI patients only.

A total of 25 of our patients fulfilled the ARDS criteria, with an overall incidence of $10.37 \%$ of total admissions during the period. This finding differs from that observed in the studies by Randolph $^{8}$ and Farias ${ }^{7}$, where the incidence of ARDS was $7.6 \%$ and $2 \%$, respectively. This elevated incidence found in our study was not surprising, as $91.83 \%$ of our population presented a chronic functional status, which is clearly identified as a risk factor for ARDS. ${ }^{16}$ Besides that, our diagnosis of ARDS was done by active search, given the prospective profile of the study.

The PALISI trial showed that $52 \%$ of patients with $\mathrm{PaO}_{2} / \mathrm{FiO}_{2}$ of 200 or less, for at least 1 day, which could correspond to transitory hypoxemia, was an indication for developing ARDS during PICU stay or represented ARDS underrecognition. ${ }^{8}$ We expected to find hypercapnia, consistent with lungprotective ventilation. ${ }^{3-6,}$ 16. However, most patients presented normo or hypocapnia, showing that we still underuse this life-saving ventilatory strategy.

In ARDS and ALI patients, risk factors for mortality and/or prolonged mechanical ventilation have been identified, which include the following: initial $\mathrm{pH}^{17,18}, \mathrm{PaO}_{2} / \mathrm{FiO}_{2}{ }^{17,19}$, tidal volumes higher than $8 \mathrm{ml} / \mathrm{kg}^{4,5,17}$, initial PEEP ${ }^{17}$, initial inspiratory pressure and oxygenation index. ${ }^{17,20}$ In our population, we also identified inspiratory pressure, $\mathrm{pH}, \mathrm{PaO}_{2} / \mathrm{FiO}_{2}$, oxygenation index at $\mathrm{TO}$ but only 
oxygenation index at T1 of MV. No difference PEEP and tidal volume between survivors and non-survivors was observed. It is possible that such difference exists but the present study had insufficient statistical power to detect it.

Mortality 28 days after admission was at around $35 \%$, well above the $1.3 \%$ expected by PELOD. Survival could be influenced by organ failure, immunocompromise and perhaps by the development of complications associated to the ventilatory strategies employed. It is also known that many models (PIM, PIM2, PRISM) underestimate mortality ${ }^{21-22}$. We used the PELOD score but could not say it underestimates mortality as well, as only a subpopulation of children admitted to the PICU was studied. Besides, this study did not aim to evaluate correlation and agreement between predicted and actual mortality by PELOD.

Specifically in "Instituto da Criança" PICU, the entire medical team is well familiar with the concept of ARDS/ALI and its treatment. Although adequate ventilator devices are available in order to implement an adequate ventilatory strategy, there is no written protocol to do so, allowing for personal variations in compliance with the strategy. It is also possible that high $\mathrm{PaCO} 2$ makes medical teams uncomfortable and that respiratory rates are elevated to compensate for the hypercapnia seen.

Many studies have tried to explain the reasons for the underuse of protective lung strategies in adult intensive care units. ${ }^{23-26}$ These includes: concerns over patient discomfort and tachypnea, hypercapnia and acidosis, worsening oxygenation, perception of contraindications to low tidal volumes, disease unrecognition ${ }^{27}$, and assistance team being uncomfortable with low tidal volumes (especially for the risk of atelectasis). 
Wolthuis and colleagues studied tidal volume use in three adult ICUs in the Netherlands to determine the effect of feedback and education concerning the use of lung protective MV. They have found that education did improve physician compliance in the use of lung protective ventilatory strategies, with a tidal volume decline within 6 months after intervention from $9.8 \mathrm{ml} / \mathrm{kg} \pm 2.0$ at baseline to $8.1 \pm 1.7 \mathrm{ml} / \mathrm{kg}^{28}$ The feedback and education program focused on lung-protective MV, with an emphasis on adjusting tidal volumes to predicted body weight (PBW).

In our study we too observed that protective lung strategies for ALI/ARDS were not fully implemented, as ventilatory settings resulting in normocapnia / hypocapnia were still being used. It is possible that written protocols and feedback orientations, as well as an education program may improve compliance with lung-protective strategies. Thus, we suggested that such strategies be implemented as an attempt to make better use of MV and to reduce mortality rates.

\section{CONCLUSIONS}

Here we showed that pressure ventilation (controlled and/or pressure support) was the ventilatory mode of choice used in this facility. Inspiratory pressures over $25 \mathrm{cmH}_{2} \mathrm{O}$ were predictive of mortality (OR 5.5), as was MV duration of longer than 7 days (OR 5.96). Initial $\mathrm{PaO}_{2} / \mathrm{FiO}_{2}$ ratio, $\mathrm{pH}$ and oxygenation index, as oxygenation index at $48 \mathrm{~h}$, were predictive of mortality. Protective lung strategies for ALI/ARDS were not fully implemented, as ventilatory settings resulting in normocapnia / hypocapnia were still being used. 
Figures

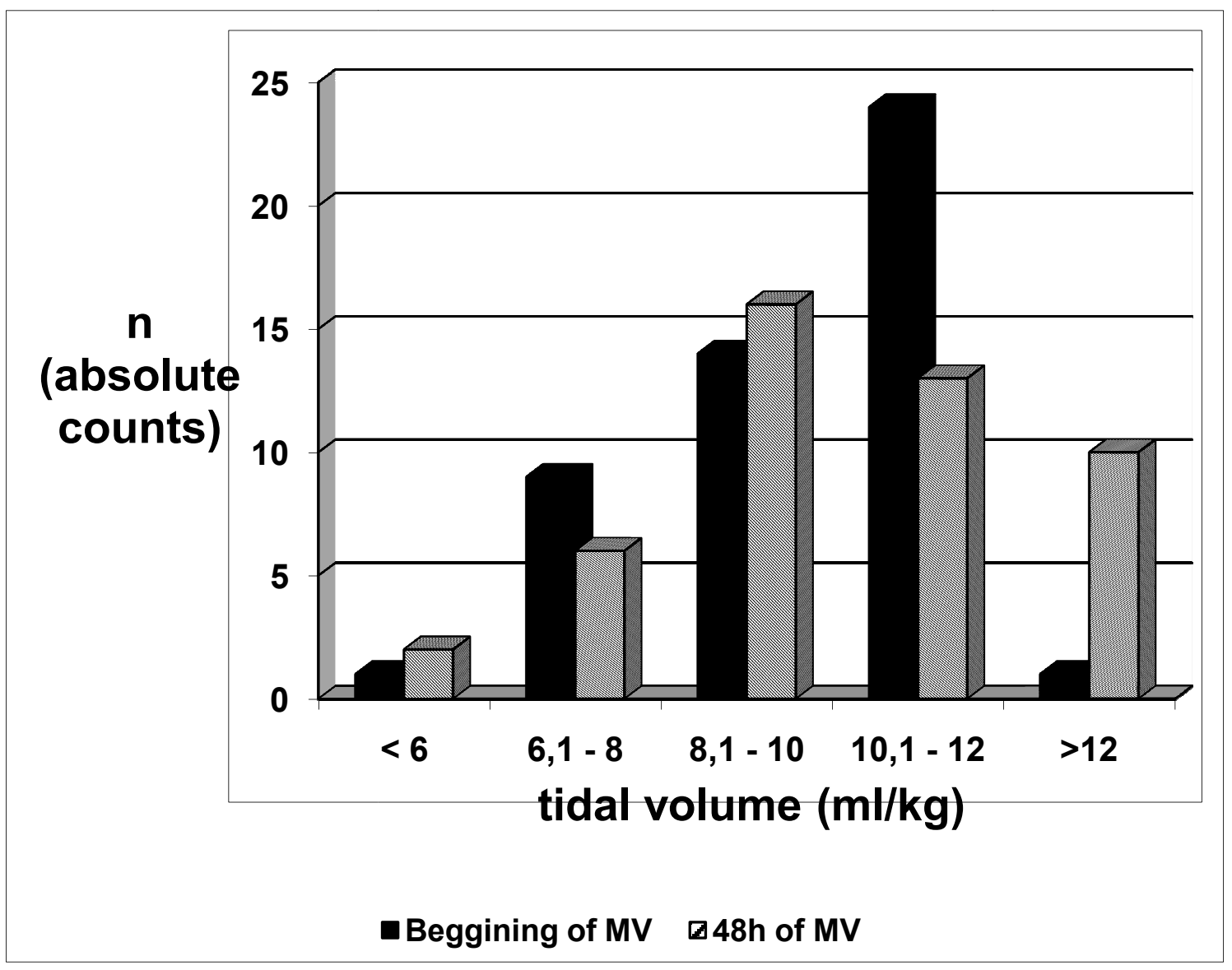

Figure 1 - Exhaled tidal volumes used $(\mathrm{ml} / \mathrm{kg})$ in children submitted for 24 hours of MV or more in a reference Brazilian PICU, from October $01^{\text {st }}, 2005$ to March $31^{\text {st }}, 2006$. 


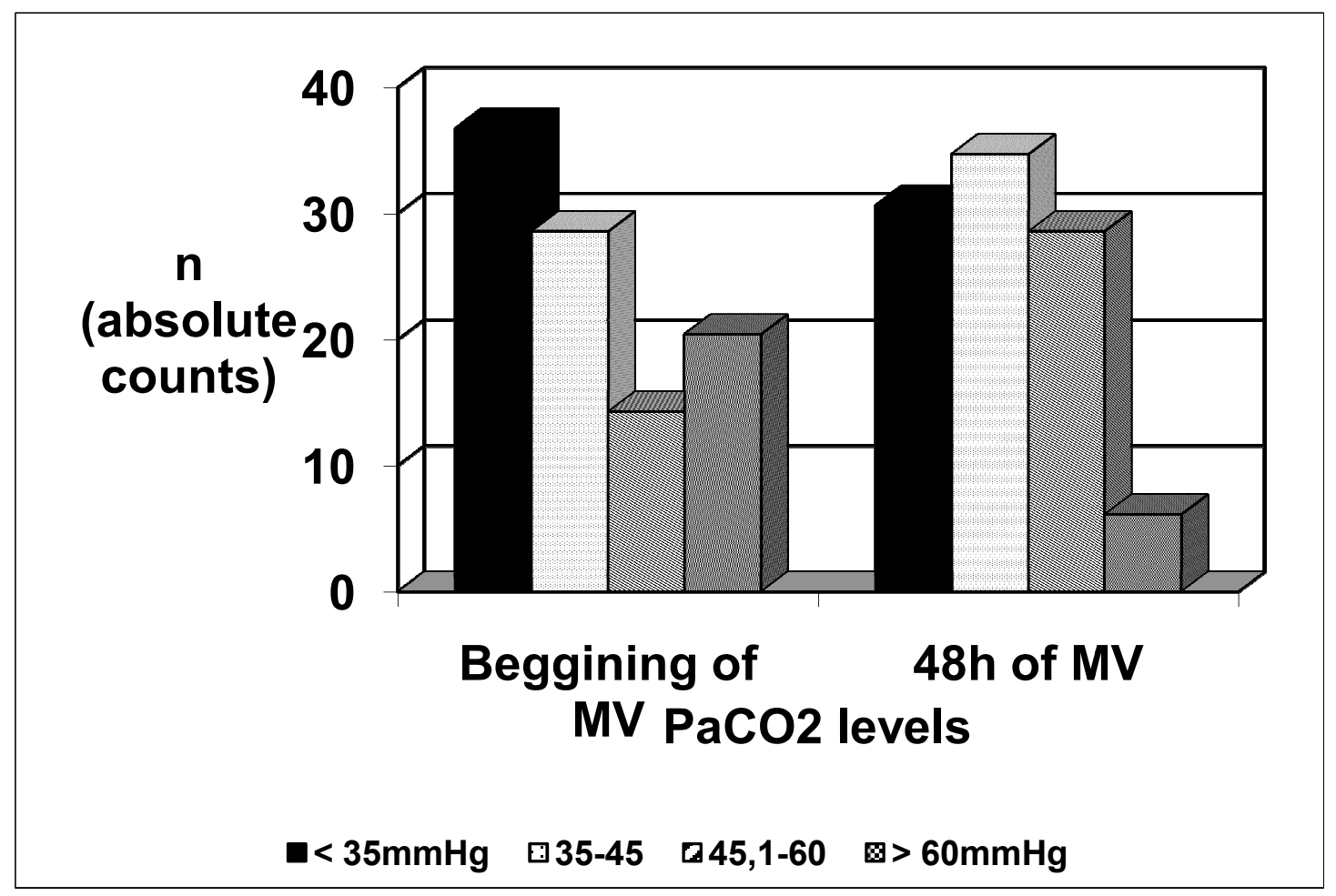

Figure $2-\mathrm{PaCO}_{2}$ levels of children submitted for 24 hours of MV or more in a reference Brazilian PICU, admitted from October 01 ${ }^{\text {st }}, 2005$ to March $31^{\text {st }}, 2006$. 
Table 1- Ventilatory settings, arterial blood gas measurements and organ dysfunctions of children submitted for 24 hours of MV or more in a reference Brazilian PICU, from October $1^{\text {st }}, 2005$ to March $31^{\text {st }}, 2006$.

\begin{tabular}{|c|c|c|}
\hline Variable & Median & P25 - P75 \\
\hline Days of MV & 6.50 & $2.50-10.50$ \\
\hline Tidal volume $(\mathrm{ml} / \mathrm{kg})$ at T0 & 10 & $9-11$ \\
\hline Tidal volume $(\mathrm{ml} / \mathrm{kg})$ at T1 & 9.5 & $8.19-10.81$ \\
\hline PEEP at T0 & 8 & $6-10$ \\
\hline PEEP at T1 & 8 & $6-10$ \\
\hline RR at T0 & 20 & $17.5-22.5$ \\
\hline $\mathrm{RR}$ at $\mathrm{T1}$ & 20 & $15.5-24.5$ \\
\hline Inspiratory pressure at T0 & 24 & $20.37-27.62$ \\
\hline Inspiratory pressure at T1 & 24 & $19.25-28.75$ \\
\hline $\mathrm{pH}$ at $\mathrm{TO}$ & 7.36 & $7.27-7.45$ \\
\hline $\mathrm{pH}$ at T1 & 7.38 & $7.32-7.49$ \\
\hline $\mathrm{PaO} 2 / \mathrm{FiO} 2$ at $\mathrm{T0}$ & 245.1 & $159.11-331.08$ \\
\hline $\mathrm{PaO} 2 / \mathrm{FiO} 2$ at $\mathrm{T} 1$ & 237.0 & $146.76-327.23$ \\
\hline Ol at T0 & 6 & $3.51-8.49$ \\
\hline Ol at T1 & 5.8 & $3.81-7.79$ \\
\hline PELOD & 11 & $6-16$ \\
\hline Organ dysfunctions at T0 & 2 & $1.5-2.5$ \\
\hline Organ dysfunction at T1 & 2 & $0.5-3.5$ \\
\hline
\end{tabular}

MV: mechanical ventilation; PEEP: positive end expiratory pressure; RR: respiratory rate; $\mathrm{PaO} 2 / \mathrm{FiO} 2$ : arterial partial pressure of oxygen / inspired fraction of oxygen ratio; OI: oxygenation index (mean airway pressure $x \mathrm{FiO} 2 \mathrm{x}$ 100 / PaO2); PELOD: Pediatric Logistic Organ Dysfunction. 
Table 2 - Risk factors for mortality and for staying more than 7 days on MV, with the respective odd ratio in each case, of children submitted for 24 hours of MV or more in a reference Brazilian PICU, admitted from October 1st, 2005 to March 31st, 2006.

\begin{tabular}{|c|c|c|}
\hline Risk factor & OR for Mortality (95\% IC) & $\begin{array}{l}\text { OR for MV above } 7 \text { days } \\
(95 \% \text { IC) }\end{array}$ \\
\hline $\begin{array}{l}\text { Initial inspiratory pressure }>25 \\
\mathrm{cmH} 20\end{array}$ & $5.5(1.53-19.71)$ & $5.96(1.69-21.03)$ \\
\hline Initial tidal volume $>8 \mathrm{ml} / \mathrm{kg}$ & $1.9(0.30-3.94)$ & $1.77(0.50-6.31)$ \\
\hline Initial $\mathrm{pH}<7.20$ & $6(1.26-28.55)$ & $1.88(0.44-8.07)$ \\
\hline Initial $\mathrm{PaO} 2 / \mathrm{FiO} 2<200$ & $2.14(0.65-7.13)$ & $2.15(0.65-7.13)$ \\
\hline Initial $\mathrm{PaO} 2 / \mathrm{FiO} 2<100$ & $10.67(1.08-105.29)$ & $6.35(0.65-61.73)$ \\
\hline Initial $\mathrm{OI}>10$ & $6.77(1.46-31.30)$ & $4.16(0.93-18.72)$ \\
\hline Initial $\mathrm{OI}>14$ & $16.91(1.83-156.62)$ & ----- \\
\hline Ol at $48 \mathrm{~h}$ of $\mathrm{MV}>10$ & $4.83(0.97-23.98)$ & ----- \\
\hline Ol at $48 \mathrm{~h}$ of $\mathrm{MV}>14$ & $11.27(1.13-112.07)$ & $4.06(0.7-23.47)$ \\
\hline
\end{tabular}




\section{REFERENCES}

1. The World Health Organization Statistical Information System (WHOSIS). http://apps.who.int/whosis/data/Search.jsp?indicators=[Indicator].[MBD].Membe rs. Accessed at 07/08/2009.

2. Benicio MHD, Cardoso MRA, Gouveia NC, Monteiro CA. [Secular trends in child respiratory diseases in S. Paulo City, Brazil (1984-1996). Rev Saude Publica. 2000;34 (Suppl 6):91-101.

3. Gattinoni L, Carlesso E, Cadringher P, Valenza F, Vagginelli F, Chiumello D. Physical and biological triggers of ventilator-induced lung injury and its prevention. Eur Respir J. 2003;22 (Suppl 47):15S-25S.

4. Amato MB, Barbas CS, Medeiros DM, Magaldi RB, Schettino GP, Lorenzi-Filho G, et al. Effect of a protective-ventilation strategy on mortality in the acute respiratory distress syndrome. N Engl J Med. 1998; 338:347-54.

5. ARDS Network Investigators. Ventilation with lower tidal volumes as compared with traditional tidal volumes for acute lung injury and the acute respiratory distress syndrome: The Acute Respiratory Distress Syndrome Network. N Engl J Med. 2000;342:1301-8.

6. Albuali $\mathrm{WH}$, Singh RN, Fraser DD, Seabrook JA, Kavanagh BP, Parshuram CS, et al. Have changes in ventilation practice improved outcome in children with acute lung injury? Pediatr Crit Care Med. 2007; 8:324 -30.

7. Farias JA, Frutos F, Esteban A, Flores JC, Retta A, Baltodano A, et al. What is the daily practice of mechanical ventilation in pediatric intensive care units? A multicenter study. Intensive Care Med. 2004;30:918-25. 
8. Randolph AG, Meert KL, O'Neil ME, Hanson JH, Luckett PM, Arnold JH, et al. The feasibility of conducting clinical trials in infants and children with acute respiratory failure. Am J Respir Crit Care Med. 2003; 167:1334-40.

9. Fedora M, Kroupova L, Kosut P, Fanta I, Hrdlicka R, Kobr J, et al. [Mechanical ventilation on paediatric intensive care units in Czech Republic]. Anasthesiol Intensivmed Notfallmed Schmerzther 2005; 40:173-8.

10. Harel Y, Niranjan V, Evans BJ. The current practice patterns of mechanical ventilation for respiratory failure in pediatric patients. Heart Lung. $1998 ; 27: 238-44$.

11. Balcells Ramirez J, Lopez-Herce Cid J, Modesto Alapont V. [Prevalence of mechanical ventilation in pediatric intensive care units in Spain]. An Pediatr (Barc). 2004;61:533-41.

12. Leteurtre S, Martinot A, Duhamel A, Proulx F, Grandbastien B, Cotting J, et al. Validation of the paediatric logistic organ dysfunction (PELOD) score: prospective, observational, multicentre study. Lancet. 2003;362 :192-7.

13. Bernard G, Artigas A, Brigham K. The American-European Consensus Conference on ARDS: definitions, mechanisms, relevant outcomes, and clinical trial coordination. Am J Respir Crit Care Med. 1994;149:818-24.

14. Cardoso MP, Bourguignon DC, Gomes MM, Saldiva PH, Pereira CR, Troster EJ. Comparison between clinical diagnoses and autopsy findings in a pediatric intensive care unit in Sao Paulo, Brazil. Pediatr Crit Care Med. $2006 ; 7: 423-7$.

15. Oliveira CF, Oliveira DS, Gottschald A, Moura J, Costa G, Ventura A, et al. ACCM/PALS haemodynamic support guidelines for paediatric septic shock: 
an outcomes comparison with and without monitoring central venous oxygen saturation. Intens Care Med. 2008; 34(6):1065-75.

16. Higgins B, Costello J, Chonghaile M, Laffey J. Permissive hypercapnia in protective lung ventilatory strategies. Paediatrics and Child Health 2007; 17(3): 94-103.

17. Erickson S, Schibler A, Numa A, Nuthall G, Yung M, Pascoe E, et al; on behalf of the Paediatric Study Group, Australian and New Zealand Intensive Care Society. Acute lung injury in pediatric intensive care in Australia and New Zealand - A prospective, multicenter, observational study. Pediatr Crit Care Med. 2007;8:317-23.

18. Flori HR, Glidden DV, Rutherford GW, Matthay MA. Pediatric acute lung injury: prospective evaluation of risk factors associated with mortality. Am $\mathrm{J}$ Respir Crit Care Med. 2005;171:995-1001.

19. Dahlem $P$, van Aalderen WMC, Hamaker ME, Dijkgraaf MGW, Bos AP. Incidence and short-term outcome of acute lung injury in mechanically ventilated children. Eur Respir J. 2003;22:980-5.

20. Trachsel D, McCrindle BW, Nakagawa S, Bohn D. Oxygenation Index Predicts Outcome in Children with Acute Hypoxemic Respiratory Failure. Am J Respir Crit Care Med. 2005;172. pp 206-211.

21. Thukral A, Lodha R, Irshad M, Arora NK. Performance of Pediatric Risk of Mortality (PRISM), Pediatric Index of Mortality (PIM), and PIM2 in a pediatric intensive care unit in a developing country. Pediatr Crit Care Med. 2006;7:356 61. 
22. Martha VF, Garcia PCR, Piva JP, Einloft PR, Bruno F, Rampon V. [Comparison of two prognostic scores (PRISM and PIM) at a pediatric intensive care unit]. J Pediatr. (Rio J). 2005;81:259-64.

23. Young MP, Manning HL, Wilson DL, Mette SA, Riker RR, Leiter JC, et al. Ventilation of patients with acute lung injury and acute respiratory distress syndrome: has new evidence changed clinical practice? Crit Care Med. $2004 ; 32: 1260-5$.

24. Kalhan R, Mikkelsen M, Dedhiya P, Christie J, Gaughan C, Lanken PN, et al. Underuse of lung protective ventilation: analysis of potential factors to explain physician behavior. Crit Care Med. 2006;34:300-6.

25. Rubenfeld GD, Cooper C, Carter G, Thompson BT, Hudson LD. Barriers to providing lung-protective ventilation to patients with acute lung injury. Crit Care Med. 2004;32:1289-93.

26. Wongsurakiat $P$, Pierson D, Rubenfeld G. Changing pattern of ventilator settings in patients without acute lung injury. Chest 2004; 126:1281-91.

27. Kneyber M, Brouwers A, Caris J, Chedamni S, Plötz F. Acute respiratory distress syndrome: is it underrecognized in the pediatric intensive care unit? Intensive Care Med (2008) 34:751-754.

28. Wolthuis EK, Korevaar JC, Spronk P, Kuiper MA, Dzoljic M, Vroom MB, et al. Feedback and education improve physician compliance in use of lungprotective mechanical ventilation. Intensive Care Med. 2005; 31:540-6. 